\title{
A catalogue of high-mass X-ray binaries ${ }^{\star}$
}

\author{
Q.Z. Liu ${ }^{1,2}$, J. van Paradijs ${ }^{2}$, and E.P.J. van den Heuvel $^{2}$ \\ 1 Purple Mountain Observatory, National Astronomical Observatories, Chinese Academy of Sciences, Nanjing 210008, PR China \\ 2 Astronomical Institute "Anton Pannekoek", University of Amsterdam, Kruislaan 403, 1098 SJ Amsterdam, The Netherlands
}

Received June 15; accepted July 19, 2000

\begin{abstract}
We present a catalogue of high-mass X-ray binaries. The catalogue is an updated version of the catalogue of van Paradijs (1995). This new catalogue contains 130 sources, 61 new high-mass X-ray binaries in addition to the 69 sources listed in van Paradijs' catalogue. Most of the new sources are identified to be Be/X-ray binaries. Some sources, however, are only tentatively identified as high-mass X-ray binaries on the basis of a transient character and/or a hard X-ray spectrum. Further identification in other wavelength bands is needed to finally determine the features of these sources.

The aim of this catalogue is to provide the reader with some basic information on the X-ray sources and their counterparts in other wavelength ranges (UV, optical, IR, radio). In cases where there is some doubt about the highmass nature of the X-ray binary this is mentioned. Some doubtful cases have not been included in the catalogue although they had been suggested by some authors to be high-mass X-ray binaries, for example GRS 1915+105 and 1WGA J1958.2+3232.
\end{abstract}

Key words: stars: massive star — stars: X-ray stars: binaries - catalogs

\section{Introduction}

High-mass X-ray binaries (HMXBs) were among the very first X-ray sources detected and optically identified almost 30 years ago. These systems consist of a compact object, mostly a magnetized neutron star (NS) (X-ray pulsar) or a black hole, orbiting a massive star. The X-ray emission in these sources is due to accretion of matter from the early-type mass-losing star by the compact companion.

Send offprint requests to: Q.Z. Liu; e-mail: qzliu@astro.uva.nl

* The catalogue and references are also available in electronic form at the CDS via anonymous

ftp to cdsarc.u-strasbg.fr (130.79.128.5) or via

http://vizier.u-strasbg.fr/cgi-bin/qcat?J/A+AS/147/25
Conventionally HMXBs can be further divided into two subgroups (van Paradijs 1983): those in which the primary is a Be star (Be/X-ray binary) and those in which the primary is a supergiant ( $\mathrm{SG} / \mathrm{X}$-ray binary).

The majority of the known high-mass X-ray binaries are $\mathrm{Be} / \mathrm{X}$-ray systems. Most Be/X-ray binaries have relatively wide orbits with moderate eccentricity and their compact companions spend most of their time far away from the disc surrounding the Be stars (van den Heuvel \& Rappaport 1987; Bhattacharya \& van den Heuvel 1991; Apparao 1994). X-ray outbursts will be expected during the time of the neutron star's periastron passage, from a low-velocity and high-density wind around Be stars, and thus collectively termed Be/X-ray transients. Their X-ray spectra are usually hard. The hard X-ray spectrum along with the transience is an important characteristic of the $\mathrm{Be} / \mathrm{X}$-ray binaries.

In the second group of HMXB systems the compact star orbits a supergiant early-type star, deep inside the highly supersonic wind. The X-ray luminosity is either powered by pure stellar wind accretion or, in the case of the brighter systems, by Roche-lobe overflow via an accretion disk.

In 1983 the number of known HMXBs was about 30 (van Paradijs 1983). By the time of the previous catalogue the number of X-ray sources associated with massive stars had increased to 69 (van Paradijs 1995). The systematic study of ROSAT X-ray sources in the SMC and the LMC (Kahabka \& Pietsch 1996; Haberl et al. 1999, 2000) and systematic programme of optical identifications (Schmidtke et al. 1994; Cowley et al. 1997; Motch et al. 1998; Stevens et al. 1999; Coe \& Orosz 2000) increased the number considerably.

In this paper we present a new catalogue, which increases the number of known HMXBs to 130, including 61 newly discovered HMXBs as well as the 69 "old" ones listed in van Paradijs' catalogue. 


\section{Description of the table}

Table 1 lists the 130 HMXBs. The format of the table is similar to that of the previous one (van Paradijs 1995) and the well-known work of Bradt \& McClintock (1983), of which the present catalogue is meant to be an update. In the table the sources are ordered according to right ascension; part of the (mainly numerical) information on a source is arranged in seven columns, below which for each source additional information is provided in the form of key words with reference numbers [in square brackets]. The columns have been arranged as follows.

In Col. 1 the first line contains the source name, with rough information on its sky location according to the convention hhmm \pm ddd. Here hh and $\mathrm{mm}$ indicate the hours and minutes of right ascension, ddd the declination in units of 0.1 degree (in a small number of cases, the coordinates shown in the name are given with more, or fewer, digits). However, for a ROSAT source the name is always given in the form of hhmm.m $\pm \mathrm{ddmm}$. The prefix J indicates a name based on J2000 coordinates. Otherwise, 1950 coordinates were used in the name. Alternative source names are given in the second line. The third line of Col. 1 lists survey catalogues and experiments in which the source was listed and detected, respectively. The following abbreviations have been used.

- A: Ariel V sky survey;

- AS: ASCA;

- B: BeppoSAX;

- C: Compton $\gamma$-ray Observatory;

- E: Einstein Observatory;

- Exo: Exosat;

- G: Ginga;

- Gr: Granat;

- H: HEAO A-1 sky survey;

- Ha: Hakucho;

- K: Kvant;

- M: MIT OSO-7 sky survey;

- OAO: Orbiting Astronomical Observatory;

- R: ROSAT;

- S: SAS 3;

- SL: Space Lab;

- T: Tenma;

- U: Uhuru sky survey;

- V: Vela-5 and -6 satellites;

- X: Rossi XTE.

In the first line of Col. 2, the source types are indicated with a letter code, as follows:

- P: X-ray pulsar;

- T: transient X-ray source;

- U: ultra-soft X-ray spectrum. These sources include black-hole candidates; some "extreme ultra-soft" (EUS) source may be white dwarf (WD) on whose surface steady nuclear burning takes place.

In the third line of Col. 2, we provide some information on the type of observation from which the source position has been derived. The following abbreviations have been used: o, optical; x, X-ray; r, radio; IR, infrared. A reference on the source position is given below the columnar information under "pos.". In addition, we give an indication of the accuracy of this position, in the form of equivalent (90 percent confidence level) error radii, but in several cases this can only be considered an approximation (e.g. when the error box is not circular). When no accuracy is quoted, it is about one arcsecond or better.

Column 3 contains in the first two lines the right ascension (RA) and declination (DEC) of the source for epoch 1950 for usual name, and for epoch 2000 for the sources with the names of J2000 coordinates. RA is given as hhmmss.s to an accurcy of $0.1 \mathrm{~s}$, DEC is given in ${ }^{\circ} \prime \prime \prime$, to an accuracy of $1^{\prime \prime}$. The third line gives the galactic longitude and latitude to an accuracy of $0.1^{\circ}$ (except for sources close to the galactic center, where these coordinates are given to $0.01^{\circ}$ ).

The first and second lines of Col. 4 give names of an optical counterpart. The third line contains a reference to a finding chart. An asterisk followed by a number or letter refers to star numbers used in the finding chart; "star" refers a star in the finding chart that has not been assigned a number or letter. Many optical counterparts have been indicated with a variable-star name, as given in the General Catalogue of Variable Stars and in recent name lists of variable stars as published regularly in the IAU Information Bulletin on Variable Stars, or a number in a well-known catalogue (e.g., HD, SAO). For X-ray sources in globular clusters, the cluster name is here given, in addition to the name of a stellar optical counterpart.

The fifth column contains some photometric information on the optical counterpart. In the first line, the apparent visual magnitude, $V$, and the color indices $B-V$, and $U-B$, are listed. The second line contains the spectral type of the optical counterpart and an estimate of the interstellar reddening, $E_{B-V}$.

In Col. 6, the average X-ray flux, or the range of observed X-ray fluxes $(2-10 \mathrm{keV}$, unless otherwise indicated), is given, in units of

$$
\begin{aligned}
1 \mu \mathrm{Jy} & =10^{-29} \mathrm{erg} \mathrm{cm}^{-2} \mathrm{~s}^{-1} \mathrm{~Hz}^{-1} \\
& =2.410^{-12} \mathrm{erg} \mathrm{cm}^{-2} \mathrm{~s}^{-1} \mathrm{keV}^{-1} .
\end{aligned}
$$

The first line in Col. 7 gives the orbital period in days. The second line contains for X-ray pulsars the pulse period, in seconds.

\section{Remarks on some individual objects}

We wish to emphasize here that some sources listed in this catalogue are still uncertain. They need to be regarded 
with caution in view of many further work necessary to be done. Some sources are tentatively classfied as massive X-ray binaries due to their X-ray transience and hard $\mathrm{X}$-ray spectra. No counterpart at other bands has been found or no pulse period has been detected, or both. Also, the compact object in some weak or soft X-ray sources may be a white dwarf instead of a neutron star. If this is the case, they should be excluded from this catalogue.

Finally, we would like to make some remarks on several individual sources. We can't make sure whether these sources belong to HMXBs or not. The following three sources are listed in our catalogue, although some doubt has been reported in the literature on the nature of the high-mass X-ray binary.

1WGA J0648.0 - 4419: The weak X-ray source with a pulsation of $13 \mathrm{~s}$ was discovered by Israel et al. (1997) from ROSAT PSPC data. They believed that the system contained a hydrogen-depleted subdwarf $\mathrm{O} 6$ star and most likely a neutron star. But Bisscheroux et al. (1997) suggested the compact companion is a white dwarf star instead of a neutron star, mainly due to the ultrasoft spectrum of the X-ray source and the very close resemblance of the X-ray spectrum and luminosity with that of the soft intermediate polars.

1E1024.0 - 5732: The source was discovered with Einstein Observatory. Caraveo et al. (1989) suggested that a highly reddened O5 star was the most likely optical counterpart, and therefore proposed the system was a HMXB. The source was listed in the previous catalogue. New optical and X-ray data, however, favour that the system contains a Wolf-Rayet star and an O-type star (Mereghetti et al. 1994; Reig 1999). The origin of the $\mathrm{X}$-rays from this source is explained by the colliding wind binary model (Reig 1999).

1E1048.1 - 5937: Although the source was listed in van Paradijs's catalogue, the system can not be confirmed to be a HMXB or a LMXB. The compact star belongs to the "anomalous" X-ray pulsars, which are thought now to be related to the magnetars. Mereghetti et al. (1998) argued that if it has a companion this star must be either a white dwarf or a helium burning star instead of a main-sequence companion.

The following two sources are not listed in our catalogue, although some authors argued that the sources are Be/X-ray binaries.

GRS 1915+105: The source was listed in van Paradijs' catalogue of low-mass X-ray binaries (LMXBs). Its compact is well accepted as a black hole candidate. Mirabel et al. (1997) argued the system was a Be/X-ray binary according to its $K$-band infrared spectra. On the other hand, most characteristics are consistent with a LMXB. Therefore, we prefer to consider the source to be a LMXB as before.

1WGA J1958.2 + 3232: Israel et al. (1998) discovered $\sim 12$ min X-ray pulsations in this source. Further spectral observations of the optical counterpart made Israel et al. (1999b) conclude that the X-ray source is a likely new accreting neutron star in a Be/X binary system. Negueruela et al. (2000), however, found the optical spectrum is dominated by emission lines from an accretion disk and argued that the observed X-ray and the optical characteristics are consistent with the object being an intermediate polar.

PSR B1259 - 63/SS 2883 is also not listed in our catalogue. The source was discovered by Johnston et al. (1992a) to be a radio pulsar in a highly eccentric orbit around the massive star SS 2883 (Johnston et al. 1992b), with an eccentricity of 0.869 . The pulsar rotation period is $47.76 \mathrm{~ms}$ and the orbital period is 1236.79 days. X-ray observations with ROSAT, ASCA and CGRO (Cominsky et al. 1994; Greiner et al. 1995; Kaspi et al. 1995; Grove et al. 1995; Tavani et al. 1996) showed X-ray luminosity of $10^{33}-10^{34}$, assuming a distance of $2 \mathrm{kpc}$. No $\mathrm{X}$-ray pulsation was detected. Optical observations of SS 2883 exhibited strong H-Balmer emissions and the star is of spectral type of about B2e, indicating its mass to be $10 M_{\odot}$ and its radius $6 R_{\odot}$ (Johnston et al. 1994). The system is the only known binary where a radio pulsar is observed to interact with gaseous material from a Be star. It may be the evolutionary missing link connecting the radio pulsars to the X-ray emitting Be binaries (for recent simple review, see Tavani \& Arons 1997).

Note added in proof: While the paper was under referee, Haberl F. and Sasaki M. (2000, A\&A 359, 573) reported that there are more than 20 new Be/X-ray binary candidates in the SMC, which are not listed in our catalogue.

Acknowledgements. QZL acknowledges the financial support from K.C. Wong fellowship of Chinese Academy of Sciences. This work is partially supported by The Netherlands Organization for Scientific Research (NWO) through Spinoza Grant 08-0 to E.P.J. van den Heuvel and by the National Project for Fundamental Research by the Ministry of Science and Technology of China (973 Project).

\section{References}

[1] Aab O.E., 1987, Bull. Spec. Aph. Obs. North Caucasus 25, 26

[2] Aab O.E., et al., 1983, SvA 27, 603

[3] Aab O.E., et al., 1984, SvA Lett. 9, 285

[4] Aab O.E., et al., 1984, SvA Lett. 10, 386

[5] Aab O.E., et al., 1984, Bull. Spec. Aph. Obs. North Caucasus 17, 1

[6] Abell G.O., Margon B., 1979, Nat 279, 701

[7] Allen D., 1977, IAU Circ. 3143

[8] Allen D.A., 1984, MNRAS 207, 45P

[9] Anderson S.F., et al., 1983, ApJ 269, 605

[10] Anderson S.F., et al., 1983, ApJ 273, 697

[11] Anderson S.F., et al., 1991, ApJ 371, 332

[12] Angelini L., et al., 1989, ApJ 346, 906 
[13] Angelini L., et al., 1998, A\&A 339, L41

[14] Antokhina E.A., Cherepashchuk A.M., 1988, SvA 31, 295

[15] Antokhina, E.A., Cherepashchuk A.M., 1988, SvA Lett. 11,4

[16] Aoki T., et al., 1992, PASJ 44, 461

[17] Apparao K.M.V., et al., 1978, Nat 271, 225

[18] Apparao K.M.V., et al., 1980, A\&A 89, 249

[19] Apparao K.M.V., 1994, Sp. Sci. Rev. 69, 255

[20] Araya R.A., Harding A.K., 1996, A\&AS 120, 183

[21] Armstrong J.T., et al., 1980, ApJ 236, L131

[22] Ash T.D., et al., 1999, MNRAS 307, 357

[23] Aslanov A.A., et al., 1987, SvA Lett. 13, 369

[24] Assadulaev S.S., Cherepashchuk A.M., 1986, SvA 30, 57

[25] Audley M.D., et al., 1996, ApJ 457, 397

[26] Balog N.I., et al., 1983, SvA 27, 310

[27] Balona L.A., 1990, MNRAS 245, 92

[28] Balona L.A., et al., 1992, A\&AS 92, 533

[29] Balucinska-church M., et al., 2000, MNRAS 311, 861

[30] Band D.L., 1989, ApJ 336, 937

[31] Band D.L., Gordon M.A., 1989, ApJ 338, 945

[32] Barr P., Van der Woerd H., 1990, ApJ 352, L41

[33] Barr P., et al., 1985, MNRAS 216, 65P

[34] Baykal A., et al., 2000, IAU Circ. 7355

[35] Becker R.H., et al., 1977, ApJ 216, L11

[36] Becklin E.E., et al., 1972, Nat 245, 302

[37] Belloni T., Hasinger G., 1990, A\&A 227, L33

[38] Belloni T., Hasinger G., 1990, A\&A 230, 103

[39] Belloni T., et al., 1993, A\&A 271, 487

[40] Belloni T., et al., 1999, ApJ 527, 345

[41] Berdnik E.V., et al., 1990, SvA Lett. 16, 472

[41a] Bergner Yu.K., et al., 1995, A\&AS 112, 221

[42] Bernacca P.L., 1984, A\&A 130, L8

[43] Bernacca P.L., Bianchi L., 1981, A\&A 94, 345

[44] Beskrovnaya N.G., 1989, SvA Lett. 14, 314

[45] Bhattacharya D., van den Heuvel E.P.J., 1991, Phys. Rep. 203, 1

[46] Bianchi L., Bernacca P.L., 1980, A\&A 89, 214

[47] Bianchi L., Pakull M., 1985, A\&A 146, 242

[48] Bignami G.F., et al., 1981, ApJ 247, L85

[49] Bisscheroux B.C., et al., 1997, A\&A 317, 815

[50] Bochkarev N.G., et al., 1988, SvA 32, 405

[51] Bohlin R.C., 1970, ApJ 162, 571

[52] Bolton C.T., 1972, Nat 235, 271

[53] Bonnet-Bidaud J.M., Chardin G., 1989, Phys. Rept. 170, 325

[53a] Bonnet-Bidaud J.M., Mouchet M., 1989, A\&A 332, L9

[54] Bonnet-Bidaud J.M., Van der Klis M., 1981, A\&A 97, 134

[55] Bonnet-Bidaud J.M., Van der Klis M., 1981, A\&A 101, 299

[56] Bonnet-Bidaud J.M., et al., 1981, A\&A 101, 184

[57] Bonsignori-Facondi S.R., et al., 1986, A\&A 166, 157

[58] Bord D.J., 1979, A\&A 77, 309

[59] Bord D.J., et al., 1976, ApJ 203, 689

[60] Borozdin K., et al., 1990, SvA Lett. 16, 345

[61] Boynton P.E., et al., 1984, ApJ 283, L53

[62] Boynton P.E., et al., 1986, ApJ 307, 545

[63] Bradt H.V., McClintock J.E., 1983, ARA\&A 21, 63

[64] Bradt H.V., et al., 1977, Nat 269, 21

[65] Braes L.L.E., Miley G.K., 1971, Nat 232, 246

[66] Braes L.L.E., Miley G.K., 1976, Nat 264, 731

[67] Branduardi G., et al., 1978, MNRAS 185, 137

[68] Brinkmann W., et al., 1989, A\&A 218, L13
[69] Brinkmann W., et al., 1991, A\&A 241, 112

[70] Brodsaya E.J., Shajn P.F., 1958, Izw. Krim. Ap. Obs. 20, 299

[71] Brucato R.J., et al., 1972, ApJ 173, L105

[72] Brucato R.J., et al., 1972, ApJ 175, L137

[73] Bruhweiler F.C., et al., 1981, ApJS 46, 255

[74] Brunet J.P., et al., 1975, A\&AS 21, 109

[75] Burderi L., et al., 1998, ApJ 498, 831

[76] Burderi L., et al., 2000, ApJ 530, 429

[77] Buscombe W., 1962, MNRAS 124, 189

[78] Buscombe W., 1980, MK Classification, 4th General Catalogue, Evanston

[79] Campana S., 1997, A\&A 320, 840

[80] Campana S., et al., 1999, A\&A 352, L91

[81] Caraveo P.A., et al., 1989, ApJ 338, 338

[82] Carpenter G.F., et al., 1977, MNRAS 179, 27P

[83] Castro-Tirado A.J., 1996, IAU Circ. 6516

[84] Chakrabarty D., et al., 1993, ApJ 403, L33

[85] Chakrabarty D., et al., 1995, ApJ 446, 826

[86] Chakrabarty D., et al., 1998, IAU Circ. 7048

[87] Chakrabarty D., et al., 1998, IAU Circ. 7062

[88] Charles P.A., et al., 1975, Ap. Lett. 16, 145

[89] Charles P.A., et al., 1983, MNRAS 202, 657

[90] Charles P.A., et al., 1996, IAU Circ. 6305

[91] Cherepashchuk A.M., Khruzina T.S., 1981, SvA 25, 697

[92] Chevalier C., Ilovaisky S.A., 1977, A\&A 59, L9

[93] Chevalier C., Ilovaisky S.A., 1978, ESO Messenger 9, 4

[94] Clark G.W., 1990, ApJ 353, 274

[95] Clark G.W., et al., 1978, ApJ 221, L37

[96] Clark G.W., et al., 1979, ApJ 227, 54

[97] Clark G.W., et al., 1988, ApJ 324, 974

[98] Clark G.W., et al., 1997, ApJ 474, L111

[99] Clark J.S., et al., 1998, MNRAS 294, 165

[99a] Clark J.S., et al., 1998, MNRAS 297, 657

[100] Clark J.S., et al., 1999, A\&A 348, 888

[100a] Clark J.S., et al., 2000, A\&A 356, 50

[101] Clarke D., 1990, A\&A 227, 151

[102] Clayton G.C., et al., 1989, MNRAS 236, 901

[103] Codina S.J., et al., 1984, A\&AS 57, 239

[104] Coe M.J., Orosz J.A., 2000, MNRAS 311, 169

[105] Coe M.J., Payne B.J., 1985, ApSS 109, 175

[106] Coe M.J., et al., 1987, MNRAS 226, 455

[107] Coe M.J., et al., 1988, MNRAS 232, 865

[108] Coe M.J., et al., 1990, MNRAS 243, 475

[109] Coe M.J., et al., 1994, MNRAS 270, L57

[110] Coe M.J., et al., 1994, A\&A 289, 784

[111] Coe M.J., et al., 1996, MNRAS 281, 333

[112] Coe M.J., et al., 1998, MNRAS 293, 43

[113] Coe M.J., et al., 2000, MNRAS 314, 290

[114] Cominsky L., Moraes F., 1991, ApJ 370, 670

[115] Cominsky L., et al., 1978, ApJ 224, 46

[115a] Cominsky L., et al., 1994, ApJ 427, 978

[116] Conti P.S., 1978, A\&A 63, 225

[117] Conti P.S., Cowley A.P., 1975, ApJ 200, 133

[118] Cook K., 1998, IAU Circ. 6860

[119] Cook M.C., et al., 1984, in "X-ray Astronomy '84", Oda M. and Giacconi R. (eds.), p. 225

[120] Cook M.C., Page C.G., 1987, MNRAS 225, 381

[121] Cook M.C., Warwick R.S., 1987, MNRAS 225, 369; 227, 661

[122] Cooke B.A., et al., 1984, ApJ 285, 258 
[123] Corbet R.H.D., 1987, in: "Physics of Be stars", Slettebak A. and Snow T.P. (eds.). Cambridge, p. 311

[124] Corbet R.H.D., Day C.S.R., 1990, MNRAS 243, 553

[124a] Corbet R.H.D., Mason K.O., 1984, A\&A 131, 385

[125] Corbet R.H.D., Peele A.G., 2000, ApJ 530, L33

[126] Corbet R.H.D., et al., 1985, MNRAS 212, 565

[127] Corbet R.H.D., et al., 1986, MNRAS 221, 961

[128] Corbet R.H.D., et al., 1986, A\&A 162, 117

[129] Corbet R.H.D., et al., 1997, IAU Circ. 6556

[130] Corbet R.H.D., et al., 1997, ApJ 476, 833

[131] Corbet R.H.D., et al., 1997, ApJ 489, L83

[132] Corbet R.H.D., et al., 1998, IAU Circ. 6803

[133] Corbet R.H.D., et al., 1999, ApJ 511, 876

[134] Corbet R.H.D., et al., 1999, ApJ 517, 956

[135] Cowley A.P., et al., 1978, AJ 83, 1619

[136] Cowley A.P., et al., 1983, ApJ 272, 118

[137] Cowley A.P., et al., 1991, ApJ 373, 228

[138] Cowley A.P., et al., 1991, ApJ 381, 526

[139] Cowley A.P., et al., 1994, ApJ 429, 826

[140] Cowley A.P., et al., 1995, PASP 107, 145

[141] Cowley A.P., et al., 1997, PASP 109, 21

[142] Coyne G.V., McConnell D.J., 1983, Vatican Obs. Publ. 1, No. 6, 73

[143] Coyne G.V., Otten L.B., 1978, Vatican Obs. Publ. 1, No. 12,257

[144] Crampton D., Hutchings J.B., 1978, IAU Circ. 3180

[145] Crampton D., Hutchings J.B., 1981, ApJ 251, 604

[146] Crampton D., et al., 1978, ApJ 223, L79

[147] Crampton D., et al., 1978, ApJ 225, L63

[148] Crampton D., et al., 1985, ApJ 299, 839

[149] Crawford I.A., 1991, A\&A 246, 210

[150] Cusumano G., et al., 1990, in Ref. [767], p. 369

[151] Cusumano G., et al., 1998, A\&A 337, 772

[152] Cusumano G., et al., 2000, ApJ 528, L25

[153] Dachs J., 1970, A\&A 5, 312

[154] Dachs J., 1972, A\&A 21, 373

[155] Dachs J., 1976, A\&A 47, 19

[156] Dachs J., et al., 1989, A\&AS 78, 487

[157] D'Amico N., et al., 1987, A\&A 180, 114

[158] Davis R., Hartmann L., 1983, ApJ 270, 671

[159] Davison P.J.N., et al., 1977, MNRAS 181, 73P

[160] Deeter J.E., et al., 1987, ApJ 314, 634

[161] Deeter J.E., et al., 1987, AJ 93, 877

[162] Deeter J.E., et al., 1989, ApJ 336, 376

[163] De Loore C., et al., 1981, A\&A 104, 150

[164] De Loore C., et al., 1984, A\&A 141, 279

[165] Dennerl K., 1995, IAU Circ. 6184

[166] Densham R.H., Charles P.A., 1982, MNRAS 201, 171

[167] Densham R.H., et al., 1983, MNRAS 205, 1117

[168] Dickey J.M., et al., 1983, ApJ 273, L71

[169] Dieters S.W., et al., 1990, IBVS 3500

[170] Doazan V., et al., 1987, A\&A 182, L25

[171] D'Odorico S., et al., 1991, Nat 353, 329

[172] Dolan J.F., Tapia S., 1984, A\&A 139, 249

[173] Dolan J.F., Tapia S., 1988, A\&A 202, 124

[174] Dolan J.F., Tapia S., 1989, ApJ 344, 830

[175] Doll H., Brinkmann W., 1987, A\&A 173, 86

[176] Dotani T., et al., 1995, IAU Circ. 6241

[177] Dower R.G., et al., 1978, Nat 273, 364

[178] Doxsey R.E., et al., 1977, Nat 269, 112

[179] Drake S.A., 1990, AJ 100, 572

[180] Drilling J.S., 1973, AJ 80, 128
[181] Dufour R.J., Duval J.E., 1975, PASP 87, 769

[182] Dupree A., et al., 1978, Nat 275, 400

[183] Dupree A., et al., 1980, ApJ 238, 969

[184] Ebisawa K., et al., 1989, PASJ 41, 519

[185] Estalella R.A., et al., 1993, A\&A 268, 178

[186] Exosat \& Einstein Data Bases

[187] Eyles C.J., et al., 1975, Nat 254, 577

[188] Fabbiano G., Schreier E.J., 1977, ApJ 214, 235

[189] Fabian A.C., et al., 1989, MNRAS 238, 729

[190] Fabregat J., et al., 1992, A\&A 259, 522

[191] Fabrika S.N., Bychkova L.V., 1990, A\&A 240, L5

[192] Fahlman G.G., Walker G.A.H., 1980, ApJ 240, 169

[193] Fahlman G.G., et al., 1985, ApJS 58, 225

[194] Falomo R., et al., 1987, MNRAS 224, 323

[195] Feast M.W., et al., 1961, MNRAS 122, 239

[196] Feinstein A., 1968, ZfA 68, 29

[197] Fejes L., 1986, A\&A 168, 69

[198] Fejes L., et al., 1988, A\&A 189, 124

[199] Fender R., et al., 1999, MNRAS 308, 473

[200] Fender R., et al., 2000, ApJ 530, L29

[201] Ferrer O.E., 1980, A\&A 84, 108

[202] Fiedler R.L., et al., 1987, AJ 94, 1244

[203] Filipovic M.D., Pietsch W., 2000, A\&A 353, 129

[204] Filippenko A.V., et al., 1988, AJ 96, 242

[205] Finger M.H., et al., 1992, IAU Circ. 5430

[206] Finger M.H., et al., 1994, IAU Circ. 5959

[207] Finger M.H., et al., 1996, A\&AS 120, 209

[208] Finger M.H., et al., 1996, ApJ 459, 288

[209] Finger M.H., et al., 1999, ApJ 517, 449

[210] Finley J.P., et al., 1994, ApJ 429, 356

[211] Fishman G.J., et al., 1991, IAU Circ. 5394

[212] Fitzgerald M.P., 1973, A\&AS 9, 297

[213] Forman W., et al., 1973, ApJ 182, L103

[214] Forman W., et al., 1976, ApJ 206, L29

[215] Forman W., et al., 1978, ApJS 38, 357

[216] Frail D.A., Hjellming R.M., 1991, AJ 101, 2126

[217] Friend D.B., Cassinelli J.P., 1986, ApJ 303, 292

[218] Frontera F., Fuligni F., 1975, ApJ 198, L105

[219] Frontera F., et al., 1987, ApJ 320, L127

[219a] Frontera F., et al., 1998, A\&A 339, L69

[220] Garcia M.B., et al., 1998, IAU Circ. 6865

[221] Garrison R.F., et al., 1977, ApJS 35, 111

[222] Geldzahler B.J., et al., 1983, ApJ 273, L65

[223] Geldzahler B.J., et al., 1989, ApJ 342, 1123

[224] Giacconi R., et al., 1971, ApJ 167, L67

[225] Giangrande A., et al., 1980, A\&AS 40, 289

[226] Gierlinski M., et al., 1997, MNRAS 288, 958

[227] Gies D.R., Bolton C.T., 1982, ApJ 260, 240

[228] Gies D.R., Bolton C.T., 1984, ApJ 276, L17

[229] Gies D.R., Bolton C.T., 1986, ApJ 304, 371 \& 389

[230] Giovannelli F., Graziali L.S., 1992, Sp. Sci. Rev. 59, 1

[231] Giovannelli F., et al., 1985, in: Multifrequency Behaviour

of Galactic Accreting Sources, Giovannelli F. (ed.), p. 284

[232] Glass I.S., 1979, MNRAS 187, 807

[233] Gonzales G., Gonzales G., 1956, Bol. Obs. Tonantzintlay Tacubaya 2, No. 15, 16

[234] Goranskij V.P., et al., 1991, SvA Lett. 17, 399

[235] Gorayo P.S., Tur N.S., 1988, Ap\&SS 145, 263

[236] Gottwald M., et al., 1986, MNRAS 222, 21P

[237] Gottwald M., et al., 1991, A\&AS 89, 367

[238] Grankin K.N., et al., 1991, SvA Lett. 17, 415

[239] Grebenev S., Sunyaev R., 1991, IAU Circ. 5354 
[240] Gregory P.C., Taylor A.R., 1978, Nat 272, 704

[241] Gregory P.C., et al., 1972, Nat 239, 440

[242] Gregory P.C., et al., 1979, AJ 84, 1030

[243] Gregory P.C., et al., 1989, ApJ 339, 1054

[243a] Greiner J., et al., 1995, ApJ 441, L43

[244] Grindlay J.E., et al., 1984, ApJ 276, 621

[245] Grindlay J.E., et al., 1984, ApJ 277, 286

[245a] Grove J.E., et al., 1995, ApJ 447, L113

[246] Gruber D.E., 1988, ApJ 328, 265

[247] Gruber D.E., Rothschild R.E., 1984, ApJ 283, 546

[248] Haberl F., 1991, ApJ 376, 245

[249] Haberl F., 1995, A\&A 296, 685

[250] Haberl F., Pietsch W., 1999, A\&A 344, 521

[251] Haberl F., White N.E., 1990, ApJ 361, 225

[252] Haberl F., et al., 1989, ApJ 343, 409

[253] Haberl F., et al., 1997, A\&A 318, 490

[254] Haberl F., et al., 1998, A\&A 335, 587

[255] Haberl F., et al., 2000, A\&AS 142, 41

[256] Haefner R., 1988, IBVS 3260

[257] Haigh N.J., et al., 1999, MNRAS 310, L21

[258] Hammerschlag-Hensberge G., 1978, A\&A 64, 399

[259] Hammerschlag-Hensberge G., Wu C.C., 1977, A\&A 56, 433

[260] Hammerschlag-Hensberge G., et al., 1979, A\&A 76, 245

[261] Hammerschlag-Hensberge G., et al., 1980, A\&A 85, 119

[262] Hammerschlag-Hensberge G., et al., 1984, ApJ 283, 249

[263] Hammerschlag-Hensberge G., et al., 1990, ApJ 352, 698

[264] Hang H.R., et al., 1999, A\&A 344, 614

[265] Hanson C.G., et al., 1989, MNRAS 240, 1P

[266] Hao J.X., et al., 1995, A\&A 291, L31

[267] Hardorp J., et al., 1959, Luminous Stars in the Northern Milky Way I. Hamberger Sternwarte-Warner and Swasey Observatory

[268] Harrison T., et al., 2000, ApJ 528, 454

[269] Hasinger G., et al., 1990, IAU Circ. 5142

[270] Heap S., Corcoran M.F., 1992, ApJ 387, 340

[271] Heemskerk M.H.M., Van Paradijs J., 1989, A\&A 223, 154

[272] Heindl W.A., et al., 1999, ApJ 521, L49

[273] Henrichs H., et al., 1982, ApJ 268, 807

[274] Hensberge G., et al., 1973, A\&A 29, 69

[275] Henson G.D., et al., 1983, ApJ 275, 247

[276] Hermsen W., et al., 1977, Nat 269, 494

[277] Herrero A., et al., 1995, A\&A 297, 556

[278] Hill R., et al., 1974, ApJ 189, L69

[279] Hill R.J., et al., 1994, ApJ 429, 192

[280] Hiltner W.A., 1977, IAU Circ. 3039

[281] Hjellming R.M., 1973, ApJ 182, L29

[282] Hjellming R.M., 1988, in: Galactic and Extragalavtic Radio Astronomy, Verschuur G.L. and Kellerman K.I. (eds.). Springer, p. 381

[283] Hjellming R.M., 1997, IAU Circ. 6750

[284] Hjellming R.M., Johnston K.J., 1981, ApJ 246, L141

[285] Hjellming R.M., Mioduszewski A.J., 1998, IAU Circ. 6857

[286] Hjellming R.M., Wade C.M., 1971, ApJ 168, L21

[287] Hjellming R.M., et al., 1998, AAS 192, 7805

[288] Hoffleit D., Jaschek C., 1982, Bright Star Catalogue, Yale [289] Holt S.S., et al., 1979, ApJ 233, 344

[290] Honeycutt R.K., Schlegel E.M., 1985, PASP 97, 300

[291] Hony S., et al., 2000, A\&A 355, 187

[292] Horaguchi T., et al., 1994, PASJ 46, 9

[293] Houk N., Cowley A.P., 1975, Michigan Catalogue of Twodimensional Spectral Types for the D stars I, Ann Arbor
[294] Howarth I.D., 1983, MNRAS 203, 801

[295] Howarth I.D., et al., 1984, MNRAS 207, 287

[296] Howe S.K., et al., 1983, ApJ 272, 678

[297] Huckle H.E., et al., 1977, MNRAS 180, 21P

[298] Hudec R., Ratz K., 1989, in Ref. [767], p. 427

[299] Hughes J.P., 1994, ApJ 427, L25

[300] Hughes J.P., Smith R.C., 1994, AJ 107, 1363

[301] Hulleman F., et al., 1998, A\&A 337, L25

[302] Humphreys R.M., Whelan J., 1975, Observatory 95, 171

[303] Hunt L.K., et al., 1994, A\&A 290, 428

[304] Hutchings J.B., 1970, MNRAS 150, 55

[305] Hutchings J.B., 1974, ApJ 192, 677

[306] Hutchings J.B., 1977, MNRAS 181, 619

[307] Hutchings J.B., 1979, PASP 91, 657

[308] Hutchings J.B., Crampton D., 1981, PASP 93, 486

[309] Hutchings J.B., Crampton D., 1981, ApJ 247, 222

[310] Hutchings J.B., Stoeckley T.R., 1977, PASP 89, 19

[311] Hutchings J.B., et al., 1975, MNRAS 170, 313

[312] Hutchings J.B., et al., 1977, ApJ 217, 186

[313] Hutchings J.B., et al., 1978, ApJ 223, 530

[314] Hutchings J.B., et al., 1978, ApJ 225, 548

[315] Hutchings J.B., et al., 1979, ApJ 229, 1079

[316] Hutchings J.B., et al., 1981, AJ 86, 871

[317] Hutchings J.B., et al., 1982, PASP 94, 541

[318] Hutchings J.B., et al., 1983, ApJ 275, L43

[319] Hutchings J.B., et al., 1984, PASP 96, 312

[320] Hutchings J.B., et al., 1985, PASP 97, 418

[321] Hutchings J.B., et al., 1987, PASP 99, 420

[322] Hutchings J.B., et al., 1987, AJ 94, 340

[323] Hynes R.I., et al., 1998, IAU Circ. 6871

[324] Ilovaisky S.A., et al., 1979, A\&A 71, L17 (Erratum A\&A $75,258)$

[325] Ilovaisky S.A., et al., 1982, A\&A 114, L7

[326] Ilovaisky S.A., et al., 1984, A\&A 140, 251

[327] Imanishi K., et al., 1998, IAU Circ. 7040

[327a] in't Zand J.J.M., et al., 2000, A\&A 361, 85

[328] Iping R.C., Petterson J.A., 1990, A\&A 239, 221

[329] Israel G.L., et al., 1997, ApJ 474, L53

[330] Israel G.L., et al., 1997, ApJ 484, L141

[331] Israel G.L., et al., 1998, MNRAS 298, 502

[332] Israel G.L., et al., 1999, IAU Circ. 7101

[333] Israel G.L., et al., 1999, A\&A 345, L1

[334] Israel G.L., et al., 2000, ApJ 531, L131

[334a] Israel G.L., et al., 2000, MNRAS 314, 87

[335] Isserstedt J., 1975, A\&AS 19, 259

[336] Ives J.C., et al., 1975, Nat 254, 578

[337] Iye M., 1986, PASJ 38, 463

[338] Iye M., Kodaiza K., 1986, PASP 97, 1186

[339] Janot-Pacheco E., et al., 1981, A\&A 99, 274

[340] Janot-Pacheco E., et al., 1982, IAU Symp. 98, 151

[341] Janot-Pacheco E., et al., 1987, A\&A 177, 91

[342] Janot-Pacheco E., et al., 1988, A\&A 202, 81

[343] Jarad M.M., et al., 1989, MNRAS 238, 1085

[344] Jaschek M., Jaschek C., 1963, PASP 75, 365

[345] Johns M., et al., 1978, IAU Circ. 3171

[346] Johnston K.J., et al., 1986, ApJ 309, 707

[347] Johnston M.D., et al., 1978, ApJ 223, L71

[348] Johnston M.D., et al., 1979, ApJ 230, L11

[349] Johnston M.D., et al., 1979, ApJ 233, 514

[350] Johnston M.D., et al., 1980, Nat 285, 26

[350a] Johnston S., et al., 1992a, MNRAS 255, 401

[350b] Johnston S., et al., 1992b, ApJ 387, L37 
[350c] Johnston S., et al., 1994, MNRAS 268, 430

[351] Jones C., et al., 1973, ApJ 181, L43

[352] Kaaret P., et al., 1999, ApJ 523, 197

[353] Kahabka P., 2000, A\&A 354, 999

[354] Kahabka P., Pietsch W., 1996, A\&A 312, 919

[355] Kahabka P., Pietsch W., 1998, IAU Circ. 6840

[356] Kahabka P., et al., 1999, A\&AS 136, 81

[357] Kallman T.R., et al., 1987, ApJ 317, 746

[358] Kamata Y., et al., 1990, PASJ 42, 785

[359] Kaper L., et al., 1990, Nat 347, 652

[360] Kaper L., et al., 1995, A\&A 300, 446

[361] Kaper L., et al., 1997, ApJ 475, L37

[361a] Kaspi V., et al., 1995, ApJ 453, 424

[362] Katz J.L., et al., 1984, AJ 89, 1604

[363] Kawai N., et al., 1989, PASJ 41, 491

[364] Kelley R.L., et al., 1981, ApJ 243, 251

[365] Kelley R.L., et al., 1983, ApJ 264, 568

[366] Kelley R.L., et al., 1983, ApJ 268, 790

[367] Kelley R.L., et al., 1983, ApJ 274, 765

[368] Kemp J.C., et al., 1983, ApJ 271, L65

[369] Kemp J.C., et al., 1986, ApJ 305, 805

[370] Kemp J.C., et al., 1987, SvA 31, 170

[371] Kendziorra E., et al., 1992, Proc. 28th Yamada Conf.

"Frontiers in X-ray Astronomy", p. 51

[372] Kendziorra E., et al., 1995, A\&A 291, L31

[373] Khruzina T.S., Cherepashchuk A.M., 1986, SvA 30, 295

[374] Khruzina T.S., Cherepashchuk A.M., 1986, SvA 30, 422

[375] Khruzina T.S., Cherepashchuk A.M., 1987, SvA 31, 180

[376] Kinugaso K., et al., 1998, ApJ 495, 435

[377] Kitamoto S., et al., 1987, PASJ 39, 259

[378] Kitamoto S., et al., 1989, PASJ 41, 81

[379] Kitamoto S., et al., 1990, PASJ 42, 85

[380] Kitamoto S., et al., 1992, ApJ 384, 263

[381] Kodaira K., et al., 1985, PASJ 37, 97

[382] Kodaira K., et al., 1985, ApJ 296, 232

[383] Koh D.T., et al., 1997, ApJ 479, 933

[384] Konig M., Maisack M., 1997, ApJ 327, L33

[385] Kopylov I.M., et al., 1986, SvA 30, 408

[386] Kopylov I.M., et al., 1987, SvA 31, 410

[387] Kouveliotou C., et al., 1992, IAU Circ. 5592

[388] Koyama K., et al., 1990, Nat 343, 148

[389] Koyama K., et al., 1990, ApJ 356, L47

[390] Koyama K., et al., 1990, PASJ 42, L59

[391] Koyama K., et al., 1991, ApJ 366, L19

[392] Koyama K., et al., 1990, ApJ 370, L77

[393] Kriss G.A., et al., 1983, ApJ 266, 806

[394] Krzeminsky W., et al., 1974, ApJ 192, L135

[395] Kuiper L., et al., 1988, A\&A 203, 79

[396] Kunjaya C., Hirata R., 1995, PASJ 47, 589

[397] Lamb R.C., et al., 1980, ApJ 239, 651

[398] Lamb R.C., et al., 1983, Nat 305, 37

[399] Lamb R.C., et al., 1999, IAU Circ. 7081

[400] Lang F.L., et al., 1981, ApJ 246, L21

[401] Lapshov I., et al., 1992, SvA Lett. 18, 12

[402] Leahy D.A., Ananth A.G., 1992, MNRAS 256, 39P

[403] Leahy D.A., Matsoka M., 1990, ApJ 355, 627

[404] Leahy D.A., et al., 1988, PASJ 40, 197

[405] Leahy D.A., et al., 1989, MNRAS 237, 269

[406] Leibowitz E.M., 1984, MNRAS 210, 279

[407] Leibowitz E.M., Mendelson H., 1992, PASP 94, 977

[408] Leibowitz E.M., et al., 1984, MNRAS 206, 751

[409] Leibowitz E.M., et al., 1984, Nat 307, 341
[410] Leisawitz D., 1988, NASA Ref. Publ. 1202

[411] Levine A., et al., 1991, ApJ 381, 101

[412] Levine A., et al., 1993, ApJ 410, 328

[413] Li F., et al., 1978, Nat 271, 37

[414] Li F., et al., 1979, ApJ 228, 893

[415] Li X.D., van den Heuvel E.P.J., 1997, A\&A 321, L25

[416] Li X.D., van den Heuvel E.P.J., 1999, ApJ 513, L45

[417] Liller W., 1973, ApJ 184, L37

[418] Ling J.C., et al., 1983, ApJ 275, 307

[419] Ling J.C., et al., 1987, ApJ 321, L117

[420] Ling J.C., et al., 1997, ApJ 484, 375

[421] Liu Q.Z., Hang H.R., 1999, A\&A 350, 855

[422] Liu Q.Z., Hang H.R., 2000, ApSS (accepted)

[423] Liu Q.Z., et al., 2000, A\&A 359, 646

[424] Lochner J.C., 1998, IAU Circ. 6858

[425] Lochner J.C., 1998, IAU Circ. 7007

[426] Lochner J.C., et al., 1989, ApJ 337, 823

[427] Lochner J.C., et al., 1991, ApJ 376, 295

[428] Lochner J.C., et al., 1998, IAU Circ. 6814

[429] Lochner J.C., et al., 1999, AAS 194, 5218

[430] Long K.S., et al., 1981, ApJ 248, 925

[431] Lucke P., 1974, ApJS 28, 73

[432] Lum K.S.K., et al., 1992, ApJS 78, 423

[433] Lund N., et al., 1991, IAU Circ. 5448

[434] Lyubimkov L.S., et al., 1997, MNRAS 286, 549

[435] Lyutyi V.M., 1985, SvA 29, 429

[436] Lyutyi V.M., et al., 1989, SvA Lett. 15, 182

[437] MacConnell D.J., 1982, A\&AS 48, 355

[438] MacConnell D.J., Coyne G.V., 1983, Vatican Obs. Pub. 2, No. 5, 63

[439] Macomb D.J., et al., 1999, ApJ 518, L99

[440] Makino F., et al., 1990, IAU Circ. 5148

[441] Makishima K., et al., 1984, PASJ 36, 679

[442] Makishima K., et al., 1987, ApJ 314, 619

[443] Makishima K., et al., 1990, ApJ 365, L59

[444] Makishima K., et al., 1990, PASP 42, 295

[445] Maraschi L., et al., 1981, ApJ 248, 1010

[446] Margon B., 1980, Ann. NY Ac. Sci. 336, 550

[447] Margon B., 1984, ARA\&A 22, 507

[448] Margon B., Anderson S.F., 1989, ApJ 347, 448

[449] Margon B., et al., 1977, ApJ 216, 811

[450] Margon B., et al., 1979, ApJ 230, L41

[451] Margon B., et al., 1979, ApJ 233, L63

[452] Margon B., et al., 1984, ApJ 281, 313

[453] Markert T.H., et al., 1977, ApJ 218, 801

[454] Markert T.H., et al., 1979, ApJS 39, 573

[455] Markert T.H., et al., 1977, ApJ 218, 801

[456] Markert T.H., et al., 1978, ApJ 224, 157

[457] Marsden D., et al., 1998, ApJ 502, L129

[458] Marshell F.E., Lochner J.C., 1998, IAU Circ. 6818

[459] Marshall N., Ricketts M.J., 1980, MNRAS 193, 7P

[460] Marshell F.E., Takeshima T., 1998, IAU Circ. 6904

[461] Marshall F.E., et al., 1997, IAU Circ. 6777

[462] Marshell F.E., et al., 1998, IAU Circ. 6828

[463] Marti J., Paredes J.M., 1995, A\&A 298, 151

[463a] Marti J., et al., 1998, A\&A 338, L71

[464] Mason K.O., et al., 1976, ApJ 207, 78

[465] Mason K.O., et al., 1978, MNRAS 184, 45P

[466] Mason K.O., et al., 1986, ApJ 309, 700

[467] Massi M., et al., 1993, A\&A 269, 249

[468] Matsuoka M., et al., 1986, MNRAS 222, 605

[469] Mazeh T., et al., 1987, ApJ 317, 824 
[470] McCray R., et al., 1984, ApJ 282, 245

[471] Meekins J.F., et al., 1984, ApJ 278, 288

[472] Mendelson H., Mazeh T., 1989, MNRAS 239, 733

[473] Mendelson H., Mazeh T., 1991, MNRAS 250, 373

[474] Mennickent R.E., Sterken C., 1997, A\&AS 121, 113

[475] Mereghetti S., et al., 1987, ApJ 312, 755

[476] Mereghetti S., et al., 1991, ApJ 366, L23

[477] Mereghetti S., et al., 1992, A\&A 263, 172

[478] Mereghetti S., et al., 1994, ApJ 424, 943

[479] Mereghetti S., et al., 1998, MNRAS 296, 689

[480] Mereghetti S., et al., 2000, A\&A 354, 567

[481] Mihara T., et al., 1991, ApJ 379, L61

[482] Mikkelsen D.R., Wallerstein G., 1974, ApJ 194, 459

[483] Mirabel I.F., et al., 1997, ApJ 477, L45

[484] Misselt K.A., 1998, PASP 110, 396

[485] Mitani K., et al., 1984, Ap\&SS 103, 345

[486] Miyamoto S., Kitamoto S., 1989, Nat 342, 773

[487] Miyamoto S., et al., 1989, Nat 336, 450

[488] Miyamoto S., et al., 1992, ApJ 391, L21

[489] Moffat A.F.J., et al., 1973, A\&A 23, 433

[490] Molnar L.A., Mauche C.W., 1986, ApJ 310, 343

[491] Molnar L.A., et al., 1984, Nat 310, 662

[492] Molnar L.A., et al., 1985, in: "Radio Stars", Hjellming

R.M. and Gibson D. (eds.). Reidel, p. 329

[493] Molnar L.A., et al., 1988, BAAS 20, 736

[494] Mook D.E., et al., 1974, PASP 86, 894

[495] Morgan W.W., et al., 1955, ApJS 2, 41

[496] Motch C., Janot-Pacheco E., 1987, A\&A 182, L55

[497] Motch C., et al., 1988, A\&A 201, 63

[498] Motch C., et al., 1991, ApJ 369, 490

[499] Motch C., et al., 1991, A\&A 246, L24

[500] Motch C., et al., 1997, A\&A 323, 853

[501] Motch C., et al., 1998, A\&AS 132, 341

[502] Mouchet M., et al., 1980, A\&A 90, 113

[503] Mourard D., et al., 1989, Nat 342, 520

[504] Murakami T., et al., 1983, ApJ 264, 563

[505] Murakami T., et al., 1986, ApJ 310, L31

[506] Murdin P., et al., 1979, MNRAS 186, 43P

[507] Murdin P., et al., 1980, MNRAS 193, 135

[508] Nagase F., 1989, PASJ 41, 1

[509] Nagase F., et al., 1982, ApJ 263, 814

[510] Nagase F., et al., 1986, PASJ 38, 547

[511] Nagase F., et al., 1991, ApJ 375, L49

[512] Nagase F., et al., 1992, ApJ 396, 147

[513] Negueruela I., 1998, A\&A 338, 505

[514] Negueruela I., 1999, MNRAS 307, 695

[515] Negueruela I., et al., 1996, A\&A 315, 160

[516] Negueruela I., et al., 1997, MNRAS 284, 859

[517] Negueruela I., et al., 2000, A\&A 354, L29

[518] Nicolet B., 1978, A\&AS 34, 1

[519] Ninkov Z., et al., 1987, ApJ 321, 425 \& 438

[520] Norton A.J., et al., 1991, MNRAS 253, 579

[521] Oda M., 1977, Sp. Sci. Rev. 20, 757

[522] Oosterbroek T., et al., 1999, A\&A 351, L33

[523] Orlandini M., et al., 1998, IAU Circ. 6868

[524] Osmer P.S., et al., 1975, ApJ 195, 705

[525] Paczynski B., 1983, ApJ 273, L81

[526] Pakull M.W., 1982, Proc. Workshop on Accreting Neutron Stars, MPE Report 177, p. 53

[527] Pakull M.W., Angebault L.P., 1986, Nat 322, 511

[528] Pakull M., Parmar A., 1981, A\&A 102, L1

[529] Pakull M., et al., 1983, A\&A 122, 79
[530] Pakull M., et al., 1985, Sp. Sci. Rev. 40, 379

[531] Paragi Z., et al., 1999, New Astron. Rev. 43, 553

[532] Paredes J.M., 1987, Rev. Mex. Astron. Astrofis. 14, 395

[533] Paredes J.M., Figueras F., 1986, A\&A 154, L30

[534] Paredes J.M., et al., 1990, A\&A 232, 377

[535] Paredes J.M., et al., 1994, A\&A 288, 519

[536] Paredes J.M., et al., 1997, A\&A 320, L25

[536a] Paredes J.M., et al., 2000, Sci 288, 2340

[537] Parkes G.E., et al., 1978, MNRAS 184, 73P

[538] Parkes G.E., et al., 1980, MNRAS 190, 537

[539] Parkes G.E., et al., 1980, MNRAS 191, 547

[540] Parmar A.N., et al., 1980, MNRAS 193, 49P

[541] Parmar A.N., et al., 1989, ApJ 338, 359 \& 373

[542] Parmar A.N., et al., 1993, A\&A 275, 227

[543] Parsignault D.R., et al., 1972, Nat. Phys. Sci. 239, 123

[544] Paul B., Rao A.R., 1998, A\&A 337, 815

[545] Paul B., et al., 2000, ApJ 528, 410

[546] Payne B.J., Coe M.J., 1987, MNRAS 225, 985

[547] Penny A.J., et al., 1973, MNRAS 163, 7P

[548] Penrods D.G., Vogt S.S., 1985, ApJ 299, 653

[549] Percy J., et al., 1981, AJ 86, 53

[550] Petre P., Gehrels N., 1993, A\&A 282, L33

[551] Phlips B.F., et al., 1996, ApJ 465, 907

[552] Pietsch W., et al., 1980, ApJ 237, 964

[553] Pietsch W., et al., 1985, Sp. Sci. Rev. 40, 371

[554] Pietsch W., et al., 1986, A\&A 163, 93

[555] Polcaro V.F., et al., 1990, A\&A 231, 354

[556] Polcaro V.F., et al., 1993, A\&A 273, L49

[557] Popper D., 1950, ApJ 111, 495

[558] Pounds K.A., et al., 1975, MNRAS 172, 473

[559] Priedhorsky W.C., Holt S.S., 1987, Sp. Sci. Rev. 45, 291

[560] Priedhorsky W.C., Holt S.S., 1987, ApJ 312, 743

[561] Priedhorsky W.C., Terrell J., 1983, Nat 303, 681

[562] Priedhorsky W.C., Terrell J., 1983, ApJ 273, 709

[563] Priedhorsky W., Terrell J., 1984, ApJ 280, 661

[564] Priedhorsky W., Terrell J., 1986, ApJ 301, 886

[565] Priedhorsky W., et al., 1983, ApJ 270, 233

[566] Primini F., et al., 1977, ApJ 217, 543

[567] Punsly B., 1999, ApJ 519, 336

[568] Rappaport S.A., et al., 1971, ApJ 168, L17

[569] Rappaport S.A., et al., 1978, ApJ 224, L1

[570] Raubenheimer B.C., 1990, A\&A 234, 172

[571] Raubenheimer B.C., Ogelman H., 1990, A\&A 230, 73

[572] Ray P.S., et al., 1997, ApJ 491, 381

[573] Raymond J.C., 1982, ApJ 258, 240

[574] Reid C.A., et al., 1980, AJ 85, 1062

[575] Reid N., et al., 1988, MNRAS 232, 53

[576] Reig P., 1999, A\&A 345, 576

[577] Reig P., Roche P., 1999, MNRAS 306, 95 \& 100

[578] Reig P., et al., 1996, A\&A 311, 879

[579] Reig P., et al., 1997, A\&A 322, 183

[580] Reig P., et al., 1998, MNRAS 301, 42

[581] Remillard R.A., Canizares C.R., 1984, ApJ 278, 761

[582] Remillard R., Levine A., 1998, IAU Circ. 6826

[583] Reynolds A.P., et al., 1992, MNRAS 256, 631

[584] Reynolds A.P., et al., 1992, MNRAS 258, 439

[584a] Ribo M., et al., 1999, A\&A 347, 518

[585] Richer G., et al., 1976, ApJ 204, L73

[586] Rickard J.J., 1974, ApJ 189, L113

[587] Ricke G.H., Lebofsky M.J., 1985, ApJ 288, 618

[588] Ricker G.R., et al., 1973, ApJ 184, 237

[589] Ricker G.R., et al., 1976, ApJ 207, 333 
[590] Ricketts M.J., et al., 1981, Sp. Sci. Rev. 30, 399

[591] Robba N.R., Warwick R.S., 1989, ApJ 346, 469

[592] Robba N.R., et al., 1992, ApJ 401, 685

[593] Robinson E.L., et al., 1998, AAS 193, 5201

[594] Roche P., et al., 1993, A\&A 270, 122

[595] Roche P., et al., 1997, A\&A 322, 139

[596] Romney J.D., et al., 1987, ApJ 321, 822

[597] Rose L.A., et al., 1979, ApJ 231, 919

[598] Rosenberg F.D., et al., 1975, Nat 256, 628

[599] Rossi C., et al., 1991, A\&A 249, L19

[600] Rossiger S., 1978, IAU Circ. 3210

[601] Rothschild R.E., Soong Y., 1987, ApJ 315, 154

[602] Rothschild R.E., et al., 1977, ApJ 213, 818

[603] Rousseau J., et al., 1978, A\&AS 31, 243

[604] Rubin B.C., et al., 1997, ApJ 488, 413

[605] Russell S.C., Dopita M.A., 1990, ApJS 74, 93

[606] Sadakane K., et al., 1984, ApJ 288, 284

[607] Safi Harb S., Ogelman H., 1996, ApJ 456, L37

[608] Salotti L., et al., 1992, A\&A 253, 145

[609] Sanduleak N., 1968, AJ 73, 246

[610] Santangel A., et al., 1998, A\&A 338, L59

[611] Saraswat P., Apparao K.M.V., 1992, ApJ 401, 678

[612] Sasaki M., et al., 2000, A\&A 143, 391

[613] Sato N., et al., 1986, ApJ 304, 241

[614] Sato N., et al., 1986, PASJ 38, 731

[615] Schalinski C.J., et al., 1995, ApJ 447, 752

[616] Schmidtke P.C., Cowley A.P., 1998, IAU Circ. 6880

[617] Schmidtke P.C., et al., 1992, ASP Conf. Ser. 32, McAlister

H.A. and Hartkopf W.I. (eds.). San Francisco, ASP, p. 386

[618] Schmidtke P.C., et al., 1994, PASP 106, 843

[619] Schmidtke P.C., et al., 1995, PASP 107, 450

[620] Schmidtke P.C., et al., 1996, PASP 108, 668

[621] Schmidtke P.C., et al., 1999, AJ 117, 927

[622] Schreier E., et al., 1972, ApJ 172, L79

[623] Schreier E., et al., 1972, ApJ 178, L71

[624] Schreier E.J., et al., 1976, ApJ 204, 539

[625] Schwartz D.A., et al., 1980, AJ 85, 549

[626] Schwartz R.A., et al., 1991, ApJ 376, 312

[627] Scott D.M., et al., 1997, ApJ 488, 831

[628] Sembay S., et al., 1990, ApJ 351, 675

[629] Seward F.D., Mitchell M., 1981, ApJ 243, 736

[630] Seward F.D., et al., 1976, MNRAS 175, 39P

[631] Seward F., et al., 1980, Nat 287, 806

[632] Seward F.D., et al., 1986, ApJ 305, 814

[633] Shafter A.W., et al., 1980, ApJ 240, 612

[634] Shakhovskaya N.I., et al., 1987, Bull. Crimean Aph. Obs. 75,110

[635] Shrader C.R., et al., 1999, ApJ 512, 920

[636] Skinner G.K., 1980, Nat 288, 141

[637] Skinner G.K., et al., 1982, Nat 297, 568

[638] Skinner G.K., et al., 1989, IAU Circ. 4850

[639] Slettebak A., 1982, ApJS 50, 55

[640] Slettebak A., 1982, ApJS 59, 769

[641] Smale A.P., Charles P.A., 1989, MNRAS 238, 595

[642] Smale A.P., et al., 1984, MNRAS 210, 855

[643] Smith D., et al., 1998, IAU Circ. 6855

[644] Smith D.A., Takeshima T., 1998, IAU Circ. 7014

[645] Smith D.M., et al., 1998, ApJ 501, L181

[645a] Smith D.M., et al., 1998, ApJ 503, 877

[646] Smithsonian Astrophysical Observatory Star Catalog, Washington DC, 1966

[647] Soffitta P., et al., 1998, ApJ 494, L203
[648] Sokolov V.V., 1987, SvA 31, 419

[649] Soria R., 1999, PASA 16, 147

[650] Southwell K.A., Charles P.A., 1996, MNRAS 281, L63

[651] Spencer R.E., 1984, MNRAS 209, 869

[652] Steele I.A., et al., 1996, A\&AS 120, 213

[653] Steele I.A., et al., 1998, MNRAS 297, L5

[654] Steiner J.E., et al., 1984, ApJ 280, 688

[655] Stella L., et al., 1985, ApJ 288, L45

[656] Stevens J.B., et al., 1997, MNRAS 288, 988

[657] Stevens J.B., et al., 1999, MNRAS 309, 421

[658] Stewart G.C., et al., 1987, MNRAS 228, 293

[659] Stickland D., et al., 1997, MNRAS 286, L21

[660] Stier M., Liller W., 1976, ApJ 206, 257

[661] Stocke J., et al., 1985, PASP 97, 126

[662] Stollberg M.T., et al., 1992, Proc. Compton Symp., St Louis

[663] Stollberg M.T., et al., 1993, IAU Circ. 5836

[664] Strom R.G., et al., 1989, Nat 337, 234

[665] Sunyaev R.A., et al., 1990, SvA Lett. 16, 55

[666] Takeshima T., Chakrabarty D., 1998, IAU Circ. 7016

[667] Takeshima T., Murakami T., 1998, IAU Circ. 7032

[668] Takeshima T., et al., 1991, PASJ 43, L43

[669] Takeshima T., et al., 1994, ApJ 436, 871

[670] Takeshima T., et al., 1998, IAU Circ. 6826

[671] Takeshima T., et al., 1998, IAU Circ. 7008

[672] Takeuchi Y., et al., 1990, PASJ 42, 287

[673] Tamura K., et al., 1992, ApJ 389, 676

[674] Tananbaum H., et al., 1972, ApJ 177, L5

[675] Tashiro M., et al., 1991, MNRAS 252, 156

[675a] Tavani M., et al., 1996, A\&AS 120, 221

[675b] Tavani M., Arons J., 1997, ApJ 477, 439

[676] Tawara Y., et al., 1989, PASJ 41, 473

[677] Taylor A.R., Gregory P.C., 1982, ApJ 255, 210

[678] Taylor A.R., Gregory P.C., 1984, ApJ 283, 273

[679] Taylor A.R., et al., 1992, ApJ 395, 268

[680] Taylor M., et al., 1995, AJ 109, 396

[681] Telting J.H., Kaper L., 1994, A\&A 284, 515

[682] Telting J.H., et al., 1998, MNRAS 296, 785

[683] Terrell J., Priedhorsky W., 1984, ApJ 285, L15

[684] Thackeray A.D., 1970, MNRAS 150, 215

[685] Thackeray A.D., et al., 1972, Mem. RAS 77, 199

[686] The P.S., 1966, Contr. Bosscha Obs., No. 35

[687] Thom C., et al., 1986, A\&A 165, L13

[688] Thomas R.M., et al., 1979, MNRAS 188, 19

[689] Tjemkes S.A., et al., 1986, A\&A 154, 77

[690] Torii K., et al., 1998, ApJ 508, 854

[691] Torii K., et al., 1999, ApJ 523, L65

[692] Treves A., et al., 1980, ApJ 242, 1114

[693] Treves A., et al., 1988, ApJ 325, 119

[694] Treves A., et al., 1988, ApJ 335, 142

[695] Treves A., et al., 1990, ApJ 364, 266

[696] Tsunemi H., 1989, PASJ 41, 453

[697] Tsunemi H., Kitamoto S., 1988, ApJ 334, L21

[698] Tuohy L.R., et al., 1988, in: "Physics of Neutron Stars and Black Holes", Tanaka Y. (ed.). Universal Academic Press, Tokyo, p. 93

[699] Tweedy R.W., et al., 1989, in Ref. [767], p. 661

[700] Ubertini P., et al., 1991, ApJ 366, 544

[701] Ubertini P., et al., 1991, ApJ 383, 263

[702] Udalski A., 1999, IAU Circ. 7105

[703] Ulmer M.P., et al., 1972, ApJ 178, L121

[704] Ulmer M.P., et al., 1973, ApJ 184, L117 
[705] Unger S.J., et al., 1992, MNRAS 256, 725

[706] Unger S.J., et al., 1998, A\&A 336, 960

[707] Unno W., et al., 1990, PASJ 42, 269

[708] Vacca W.D., et al., 1986, MNRAS 220, 339

[709] Van Amerongen S.F., et al., 1986, IBVS 2901

[710] Van den Heuvel E.P.J. and Rappaport S.A., 1987, in: "Physics of Be stars", Slettebak A. and Snow T.P. (eds.). Cambridge, p. 291

[711] Van der Klis M., Bonnet-Bidaud J.M., 1984, A\&A 135, 155

[712] Van der Klis M., Bonnet-Bidaud J.M., 1989, A\&A 214, 203

[713] Van der Klis M., Jansen F., 1984, Nat 313, 768

[714] Van der Klis M., et al., 1982, A\&A 106, 339

[715] Van der Klis M., et al., 1983, MNRAS 203, 279

[716] Van der Klis M., et al., 1985, A\&A 151, 322

[717] Van Genderen A.M., 1981, A\&A 96, 82

[718] Van Kerkwijk M., et al., 1989, A\&A 209, 173

[719] Van Kerkwijk M., et al., 1992, Nat 355, 703

[720] Van Kerkwijk M., et al., 1995, A\&A 303, 483

[721] Van Oijen J.G.J., 1989, A\&A 217, 115

[722] Van Paradijs J., 1983, in: "Accretion Driven Stellar X-ray Sources", Lewin W.H.G. and van den Heuvel E.P.J. (eds.). Cambridge, p. 189

[723] Van Paradijs J., 1991, in: "Neutron Stars, Theory and Observation", Ventura J. and Pines D. (eds.). Kluwer, p. 289

[724] Van Paradijs J., 1995, in: "X-ray Binaries", Lewin W.H.G., Van Paradijs J. and van den Heuvel E.P.J. (eds.). Cambridge, p. 536

[725] Van Paradijs J., Kuiper L., 1984, A\&A 138, 71

[726] Van Paradijs J., et al., 1977, A\&AS 30, 195

[727] Van Paradijs J., et al., 1983, A\&A 124, 294

[728] Van Paradijs J., et al., 1984, A\&AS 55, 7

[729] Van Paradijs J., et al., 1984, MNRAS 210, 863

[730] Van Paradijs J., et al., 1987, A\&A 184, 201

[731] Vermeulen R., 1989, Ph.D. Thesis, Univ. Leiden

[732] Vermeulen R., et al., 1987, Nat 328, 309

[733] Vestrand W., et al., 1997, ApJ 483, L49

[734] Vidal N.V., 1973, ApJ 186, L81

[735] Vikhlinin A., et al., 1992, IAU Circ. 5576

[736] Vikhlinin A., et al., 1994, ApJ 424, 395

[737] Vrtilek S.D., et al., 1991, ApJS 76, 1127

[738] Vrtilek S.D., et al., 1997, ApJ 490, 377

[739] Wackerling L.R., 1970, Mem RAS 73, 153

[740] Wagner R.M., et al., 1986, ApJ 308, 152

[741] Wagner R.M., et al., 1989, ApJ 346, 971

[742] Wang Z.R., et al., 1990, A\&A 240, 98

[743] Warwick R.S., et al., 1981, MNRAS 197, 865

[744] Warwick R.S., et al., 1988, MNRAS 232, 551
[745] Waters L.B.F.M., 1989, in Ref. [767], p. 25

[746] Waters L.B.F.M., et al., 1988, A\&A 198, 200

[747] Waters L.B.F.M., et al., 1989, A\&A 213, L19

[748] Watson M.G., et al., 1981, MNRAS 195, 197

[749] Watson M.G., et al., 1982, MNRAS 199, 915

[750] Watson M.G., et al., 1983, ApJ 273, 688

[751] Watson M.G., et al., 1986, MNRAS 222, 261

[752] Webster B.L., Murdin P., 1971, Nat 235, 37

[753] Webster B.L., et al., 1972, Nat PS 240, 183

[754] Weisskopf M.C., et al., 1984, ApJ 278, 711

[755] Wheaton W.A., et al., 1975, IAU Circ. 2761

[756] White N.E., 1978, Nat 271, 38

[757] White N.E., Carpenter G.F., 1978, MNRAS 183, 11P

[758] White N.E., Holt S.S., 1982, ApJ 257, 318

[759] White N.E., Marshall F.E., 1984, ApJ 281, 354

[760] White N.E., Swank J.H., 1984, ApJ 287, 856

[761] White N.E., et al., 1976, MNRAS 176, 201

[762] White N.E., et al., 1978, Nat 274, 664

[763] White N.E., et al., 1980, ApJ 239, 655

[764] White N.E., et al., 1982, ApJ 263, 277

[765] White N.E., et al., 1984, ApJ 270, 711

[766] White N.E., et al., 1988, ApJ 324, 363

[767] White N.E., et al., 1989, Proc. 23rd ESLAB Symp. (ESA SP-296)

[768] White N.E., et al., 1994, IAU Circ. 6100

[769] Whitehorne M.L., 1989, JRAS Canada 83, 277

[770] Whitlock L., et al., 1989, ApJ 338, 381

[771] Whitlock L., et al., 1989, ApJ 344, 371

[772] Wickramasinghe D.T., et al., 1974, ApJ 188, 167

[773] Wilson C.A., et al., 1992, Proc. Compton Symp., St Louis

[774] Wilson C.A., et al., 1997, ApJ 479, 388

[775] Wilson C.A., et al., 1998, ApJ 499, 820

[776] Wilson C.A., et al., 1999, ApJ 511, 367

[777] Wilson R.B., et al., 1995, IAU Circ. 6207

[778] Wolff S.C., Morrisson N.D., 1974, ApJ 187, 69

[779] Wolinski K.G., et al., 1996, ApJ 457, 859

[780] Woo J.W., et al., 1996, ApJ 467, 811

[781] Wood K.S., et al., 1984, ApJS 56, 507

[782] Wood K.S., et al., 1991, ApJ 379, 295

[783] Wu C.C., et al., 1982, PASP 94, 149

[784] Wu C.C., et al., 1983, PASP 95, 391

[785] Yamauchi S., 1990, PASJ 42, L53

[786] Yamauchi S., et al., 1995, PASJ 47, 189

[787] Yang S., et al., 1988, PASP 100, 233

[788] Yokogawa J., Koyama K., 1998, IAU Circ. 6853

[789] Yokogawa J., Koyama K., 1998, IAU Circ. 7028

[790] Yokogawa J., et al., 1999, PASJ 51, 547

[791] Zamanov R.K., et al., 1999, A\&A 351, 543

[792] Zhang S.N., et al., 1996, A\&AS 120, 227

[793] Zwitter T., et al., 1989, Fund Cosm. Phys. 13, 309 
Table 1. High-mass X-ray binaries

\begin{tabular}{|c|c|c|c|c|c|c|}
\hline Name(s) & $\begin{array}{l}\text { type } \\
\text { Pos. }\end{array}$ & $\begin{array}{l}\mathrm{RA} \\
\mathrm{DEC} \\
l^{\mathrm{II}}, b^{\mathrm{II}} \\
\end{array}$ & $\begin{array}{l}\text { Opt. Ctp. } \\
{[\mathrm{FC}]}\end{array}$ & $\begin{array}{l}V, B-V, U-B \\
\text { Sp. type, } E_{B-V}\end{array}$ & $\begin{array}{l}F_{x} \\
\mu \mathrm{Jy}\end{array}$ & $\begin{array}{l}P_{\text {orb }}(\mathrm{d}) \\
P_{\text {pulse }}(\mathrm{s})\end{array}$ \\
\hline J0032.9- 7348 & & $\begin{array}{l}003256.1 \\
-734819\end{array}$ & ${ }^{*} 1$ & $\begin{array}{l}15.3,--,-- \\
\text { Be, - - }\end{array}$ & 0.2 & \\
\hline $\mathrm{R}$ & $\mathrm{x} 12.9^{\prime \prime}$ & $304.7,-43.2$ & {$[657]$} & {$[354]$} & {$[354]$} & \\
\hline
\end{tabular}

Pos.: [255]; in SMC: [354]; very strong emission lines: [657]; another early-type star within error circle: [657].

\begin{tabular}{lllll}
\hline J0049-732 & P & 004929.6 & 0.04 & \\
& & -731056 & & 9.1321 \\
As & $\times 5.5^{\prime \prime}$ & $303.1,-43.9$ & {$[327]$} & {$[327]$}
\end{tabular}

Pos.: [255]; in SMC: [327]; likely a Be: [255].

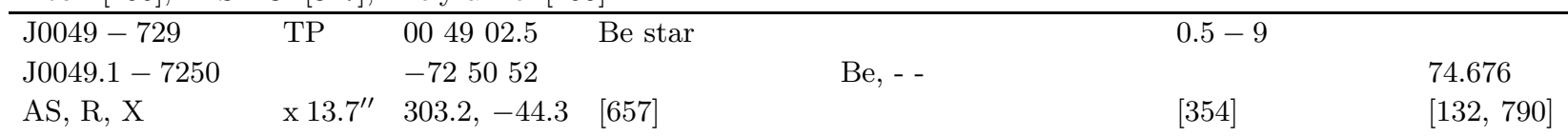

Pos.: [255, 355]; in SMC: [354]; two Be stars within error circle: [104]; high variability: [355, 354].

\begin{tabular}{|c|c|c|c|c|c|}
\hline $0050-727$ & $\mathrm{~T}$ & 005019.5 & $* 4$ & $\sim 14,-0.3,-1.0$ & $<1-5$ \\
\hline SMC X-3 & & $-7242 \quad 24$ & & O9 III-Ve, 0.03 & \\
\hline $\mathrm{A}, \mathrm{H}, \mathrm{S}, \mathrm{X}$ & o $3^{\prime \prime}$ & $302.9,-44.7$ & [95] & {$[7,146]$} & {$[95]$} \\
\hline
\end{tabular}

Pos.: [63]; transient: [95, 96]; projected rotational velocity $v_{\mathrm{r}} \sin i \sim 200 \mathrm{~km} \mathrm{~s}^{-1}:$ [146].

\begin{tabular}{lllllll}
\hline J0050.7-7316 & $\mathrm{P}$ & 005044.7 & Be star & $15.44,-0.03,-0.95$ & 0.16 & 1.416 \\
J0051-733 & & -731605 & & Be, - & $(0.7-10 \mathrm{keV})$ & 323 \\
R, AS & o $1^{\prime \prime}$ & $303.0,-43.8$ & {$[141]$} & {$[104,141]$} & {$[788]$} & {$[104,788]$}
\end{tabular}

Pos.: [104]; in SMC: [141]; orbital period 0.708 days: [118, 616]; orbital solution: [104].

\begin{tabular}{lllllll}
\hline J0051-722 & TP & 005055.8 & Be star & $14.9,--,--$ & 2.6 & 120 \\
& & -721355 & & Be & & 91.1 \\
X, R, B & $\mathrm{x} 10^{\prime \prime}$ & $303.0,-44.9$ & {$[657]$} & {$[657]$} & {$[424]$} & {$[334,461]$}
\end{tabular}

Pos.: [132]; in SMC: [461]; X-ray pulsation: [132, 334, 424]; strong emission lines: [657]; opt. spectrum: [657].

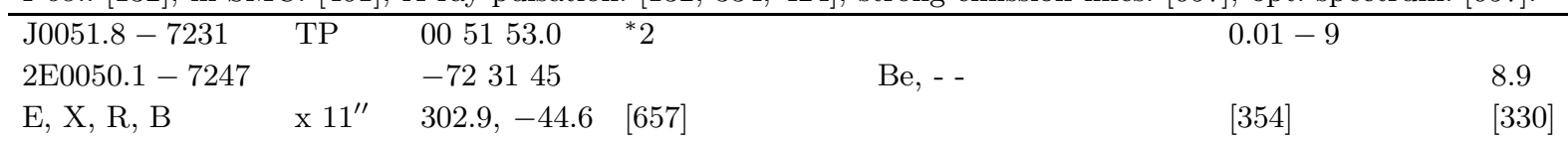

Pos.: [354]; in SMC: [354]; X-ray activity: [354]; neither AV111 nor star 1 is the opt. counterpart: [657].

\begin{tabular}{lllll}
\hline J0051.9-7311 & T & 005151.4 & B $[\mathrm{e}]$ star & $14.4,--,-$ \\
SMC 25 & & -731038 & B $[\mathrm{e}],--$ \\
R & x & $302.9,-43.9$ & {$[141]$} & {$[141]$}
\end{tabular}

Pos.: [141]; unassociated with the supernova remnent DEM 60: [141].

\begin{tabular}{lllllll}
\hline J0052.1-7319 & TP & 005213.9 & SMC SC6 99923 & $14.667,-0.005,--$ & $0.024-4.7$ & 15.3 \\
& & -731913 & & Be, - & \\
E, X, R & $\mathrm{x} 1.9^{\prime \prime}$ & $302.8,-43.8$ & {$[702]$} & {$[702,332]$} & {$[399]$} & {$[399]$}
\end{tabular}

Pos.: [255]; in SMC: [399]; highly variable X-rays: [353]; strong H $\alpha$ emis. line: [332]; $P_{\text {orb }}$ likely between $600-700$ d: [702].

\begin{tabular}{llllll}
\hline J0052.9-7158 & TU & 005254.0 & Be star & $15.46,-0.07,-0.99$ & 2 \\
SMC 32 & & -715808 & & Be, -- & $(0.15-2.4 \mathrm{keV})$ \\
R & $\mathrm{x}$ & $302.8,-45.1$ & {$[141]$} & {$[141]$} & {$[141]$}
\end{tabular}

Pos.: [141]; unlikely the supernova remnant IKT4: [141]; highly variable in X-rays: [141]; trans. supersoft source: [141]. 
Table 1. continued

\begin{tabular}{lllllll}
\hline Name(s) & type & RA & Opt. Ctp. & $V, B-V, U-B$ & $F_{x}$ & $\begin{array}{l}P_{\text {orb }}(\mathrm{d}) \\
P_{\text {pulse }}(\mathrm{s})\end{array}$ \\
& & DEC & & Sp. type, $E_{B-V}$ & $\mu$ Jy & \\
& Pos. & $l^{\mathrm{II}}, b^{\mathrm{II}}$ & {$[\mathrm{FC}]$} & & \\
\hline $0053-739$ & $\mathrm{~T}$ & 005253.1 & ${ }^{*} 5$ & $16.0,-0.3,-0.5$ & $<1-7$ \\
SMC X-2 & & -735719 & & B 1.5 Ve, 0.03 & \\
A, S & $\mathrm{o}$ & $302.6,-43.4$ & {$[95]$} & {$[506]$} & {$[95]$}
\end{tabular}

Pos.: [506]; transient: [95, 96]; southern component of a close pair: [506]; $v_{\mathrm{r}} \sin i \sim 200 \mathrm{~km} \mathrm{~s}^{-1}:[146]$.

\begin{tabular}{lllll}
\hline $0053+604$ & 005340.3 & $\gamma$ Cas & $1.6-3.0,-0.15,-1.08$ & $5-11$ \\
& +602647 & & B0.5 Ve, 0.05 & \\
$\mathrm{U}, \mathrm{A}, \mathrm{H}, \mathrm{S}, \mathrm{AS}, \mathrm{B}$ & o & $123.6,-2.1$ & {$[177]$} & {$[489]$}
\end{tabular}

Pos.: [63]; X-ray spectrum: [219, 505, 542]; UV observations: [51, 170, 261, 273, 455]; variable Be star: [235, 456, 739, 769]; radial-velocity variations: [343]; $v_{\mathrm{r}} \sin i \sim 300-500 \mathrm{~km} \mathrm{~s}^{-1}$ : [304, 310, 489]; system velocity: [721]; comparison with X Per: [489, 764]; radio/mm obs.: [179, 747]; polarimetry: [101]; optical interferometry H $\alpha$ envelope: [503, 687]; non-radial pulsations: [787]; wind structure: [746]; long-term periodic variability in UV absorption: [681]; a WD sys.: [249]; multiwavelength obs.: [292, 645a]; ISO IR spe. and envelope structure: [291].

\begin{tabular}{|c|c|c|c|c|c|}
\hline J0053.8 - 7226 & $\mathrm{TP}$ & 005355.0 & & 1.4 & 139 \\
\hline $0053-724$ & & -722647 & $\mathrm{~B} 1 \mathrm{~V}$ & & 46.63 \\
\hline $\mathrm{X}, \mathrm{R}$ & $\mathrm{x} 10^{\prime \prime}$ & $302.7,-44.7$ & & {$[132]$} & {$[132,425]$} \\
\hline
\end{tabular}

Pos.: [132]; in SMC: [132]; X-ray pulsations: [132, 425]; possibly a Be star [425]; spectral type: see [513].

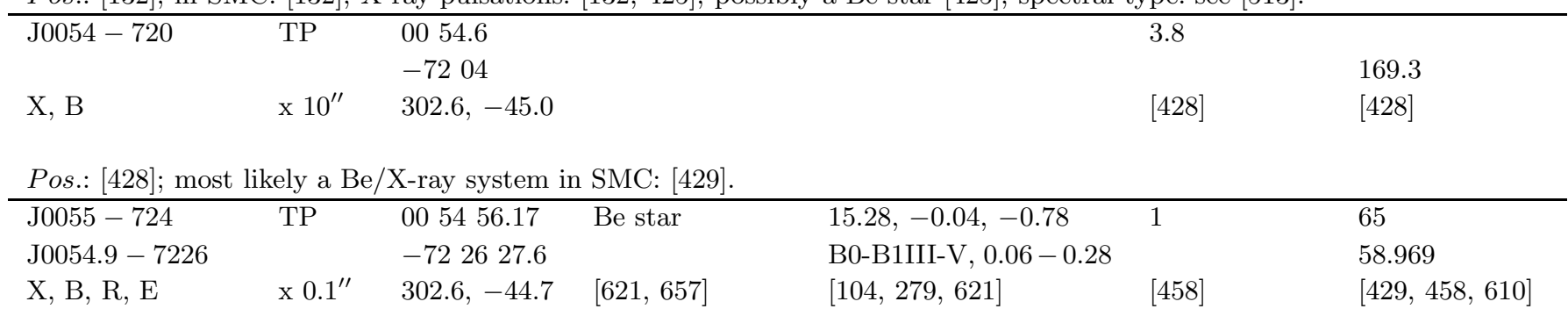

Pos.: [104]; in SMC: [458]; orbital period 14.26 days: [104]; very strong emission lines: [657]; consistent with Einstein source $2 \mathrm{E} 0053.2-7242:[610]$

\begin{tabular}{lllll}
\hline J0058-72.0 & $\mathrm{P}$ & 005748.4 & 0.1 & \\
J0058-7203 & & -720242 & $(0.7-10 \mathrm{keV})$ & 280.4 \\
AS, R & $\mathrm{x} 7.9^{\prime \prime}$ & $302.2,-45.0$ & {$[788]$} & {$[788]$}
\end{tabular}

Pos.: [255]; HMXB in SMC (?): [255].

\begin{tabular}{|c|c|c|c|c|c|c|}
\hline J0058.2 - 7231 & $\mathrm{x}$ & $\begin{array}{l}005812.7 \\
-723045 \\
302.2, \quad-44.6\end{array}$ & $\begin{array}{l}\text { Be star } \\
{[621]}\end{array}$ & $\begin{array}{l}15.0,--,-- \\
\text { Be, - - } \\
{[621]}\end{array}$ & & \\
\hline \multicolumn{7}{|c|}{ Pos.: [621]; very weak X-ray emission in SMC: [621]. } \\
\hline J0059.2-7138 & TUP & $\begin{array}{l}005911.3 \\
-713845\end{array}$ & Be star & $\begin{array}{l}14.1,0.11,-- \\
\text { B1IIIe, } 0.03\end{array}$ & $\begin{array}{l}3 \\
(0.2-2 \mathrm{keV})\end{array}$ & $\begin{array}{l}2.7632 \\
{[299]}\end{array}$ \\
\hline
\end{tabular}

Pos.: [255]; in SMC: [299]; strong H $\alpha$ emis. line: [299]; two components X-ray spe.: [299]; extremely ultrasoft X-ray sp.: [299].

$\begin{array}{llllll}\text { J0101.0-7206 } & \text { T } & 010101.1 & \text { Be star } & & 0.1 \\ & & -720657\end{array}$

Pos.: [354]; in SMC: [354]; very strong H $\alpha$ emission line: [657]. 
Table 1. continued

\begin{tabular}{lllllll}
\hline Name(s) & type & RA & Opt. Ctp. & $V, B-V, U-B$ & $\begin{array}{l}F_{x} \\
\mu \mathrm{Jy}\end{array}$ & $\begin{array}{l}P_{\text {orb }}(\mathrm{d}) \\
P_{\text {pulse }}(\mathrm{s})\end{array}$ \\
& & DEC & Sp. type, $E_{B-V}$ & & \\
& Pos. & $l^{\mathrm{II}}, b^{\mathrm{II}}$ & {$[\mathrm{FC}]$} & & & \\
\hline J0103-722 & $\mathrm{P}$ & 010313.9 & Be star & $14.8,0.089,--$ & $0.01-0.1$ \\
J0103.2-7209 & & -720914.0 & & O9-B1(III-V), 0.23 & $(0.2-2 \mathrm{keV})$ & 345.2 \\
R, A, B & o $0.1^{\prime \prime}$ & $301.6,-44.9$ & {$[300]$} & {$[104,300]$} & {$[300,334]$} & {$[334]$}
\end{tabular}

Pos.: [104]; in SMC: [334]; consistent with the known supernova remnant SNR 0101 - 724 and the Einstein source 1E $0101.5-7225$ : [300]; strong $\mathrm{H} \alpha$ activity in the BeppoSAX error circle: [334].

$\begin{array}{llllll}0103-762 & \mathrm{~T} & 010745.1 & \text { Be star } & 17,--,-- & 2.3 \\ 0107-750 & & -750038 & & \text { Be, } 0.03 & \\ \text { H, V }, \mathrm{R} & \mathrm{o} & 301.9,-41.1 & {[698]} & {[781]}\end{array}$

Pos.: [698]; variable X-ray source: [698]; member SMC: [698].

\begin{tabular}{|c|c|c|c|c|}
\hline J0105 - 722 & $\mathrm{TP}$ & 010508.9 & 0.1 & \\
\hline J0105.1 - 7211 & & -721144 & & 3.343 \\
\hline $\mathrm{AS}, \mathrm{R}$ & x $6.6^{\prime \prime}$ & $301.4,-44.9$ & [203] & {$[789]$} \\
\hline
\end{tabular}

Pos.: [255]; in SMC: [789]; containing six sources: [203]; likely a Be/X-ray binary: [203]; multiwavelenth obs.: [203].

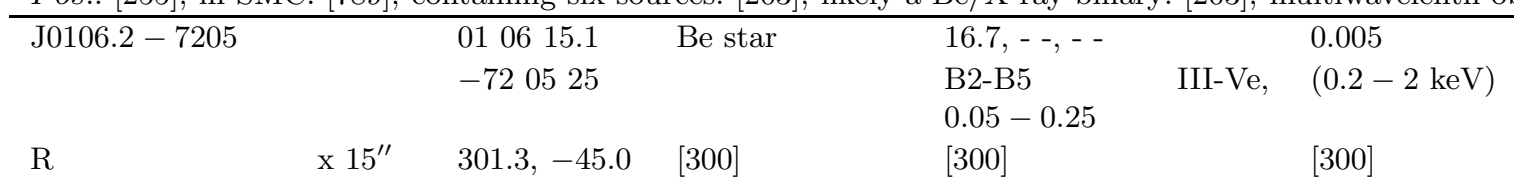

Pos.: [356]; consistent with the known supernova remnant SNR $0104-72.3$ in SMC: [300]; opt/IR obs.: [605].

\begin{tabular}{lllllll}
\hline J0111.2-7317 & TP & 011108.4 & Be star & $15.32,0.08,--$ & 15 & 31.0294 \\
& & -731646 & & B0-2III-Ve, - & \\
X, AS, C & o 1" & $301.0,-43.7$ & {$[113]$} & {$[113]$} & {$[87]$} & {$[86]$}
\end{tabular}

Pos.: [113]; in SMC: [86]; optical outbursts: [113]; very strong emission lines: [332].

\begin{tabular}{|c|c|c|c|c|c|c|}
\hline $0114+650$ & $\mathrm{P}$ & 011441.8 & V662 Cas & $11.0,1.2,0.1$ & 4 & 11.6 \\
\hline & & +650132 & $\mathrm{LS} \mathrm{I}+65^{\circ} 010$ & B0.5 Ib, 1.4 & & 10008 \\
\hline $\mathrm{A}, \mathrm{S}, \mathrm{X}$ & о $3^{\prime \prime}$ & $125.7,+2.6$ & {$[177]$} & {$[3,148]$} & [63] & {$[148,210,785]$} \\
\hline
\end{tabular}

Pos.: [63]; X-rays: [326, 619, 785]; the longest pulsation period: [133]; opt. spectra: [3, 148, 446, 421]; $v_{\mathrm{r}} \sin i \sim 45 \mathrm{~km} \mathrm{~s}{ }^{-1}$ : [3]; opt. polarimetry: [44]; review multi-wavelength behaviour: [231]; a magnetar: [416]; astrophys. parameters: [578]; opt. photometry: [680].

\begin{tabular}{lllllll}
\hline $0115+634$ & TP & 011513.8 & V635 Cas & $14.5-16.3,1.4,0.3$ & $<2-350$ & 24.3 \\
& & +632838 & & B0.2V, 1.7 & & 3.61 \\
U, M, H, S, C, X & o & $125.9,+1.0$ & {$[347]$} & {$[345,473,513]$} & {$[63]$} & {$[508,569]$}
\end{tabular}

Pos.: [347]; X-ray outbursts: [559, 590, 597, 673, 697]; recurrence time 3 yr (?): [770]; X-ray spectrum: [590, 765]; X-ray cyclotron line: [272, 511, 673]; opt. pre-outburst behaviour: [393, 473]; long-term opt. phot.: [473]; opt. spectra: [309, 706]; $v_{\mathrm{r}} \sin i \sim 365 \mathrm{~km} \mathrm{~s}^{-1}:$ [309]; multiwavelength obs. during an outburst: [516].

$\begin{array}{lllllll}0115-737 & \mathrm{P} & 011545.6 & \text { Sk } 160 & 13.3,-0.14,-0.98 & 0.5-57 & 3.89 \\ \text { SMC X-1 } & & -734222 & & \text { B0 Ib, 0.03 } & 0.71 \\ \text { U, M, A } & \text { o } 3^{\prime \prime} & 300.4,-43.6 & {[609]} & {[312,753]} & {[63]} & {[508,566,623]}\end{array}$

Pos.: [95]; highly variable X-ray source: [629]; pulse timing \& X-ray orbit: [566]; aperiodic X-ray variability: [11]; long-term X-ray obs.: [54, 247]; X-ray sp. [762, 765]; opt. lt crv. \& system parameters: [375, 417, 689, 725]; opt. spectrum \& orbit: [312, $584] ; v_{\mathrm{r}} \sin i \sim 200 \mathrm{~km} \mathrm{~s}^{-1}:$ [312]; UV obs.: [56, 262, 714]; magnetic field strength: [415]; orbital decay: [412]. 
Table 1. continued

\begin{tabular}{|c|c|c|c|c|c|c|}
\hline Name(s) & $\begin{array}{l}\text { type } \\
\text { Pos. }\end{array}$ & $\begin{array}{l}\mathrm{RA} \\
\mathrm{DEC} \\
l^{\mathrm{II}}, b^{\mathrm{II}}\end{array}$ & Opt. Ctp. & $\begin{array}{l}V, B-V, U-B \\
\text { Sp. type, } E_{B-V}\end{array}$ & $\begin{array}{l}F_{x} \\
\mu \mathrm{Jy}\end{array}$ & $\begin{array}{l}P_{\text {orb }}(\mathrm{d}) \\
P_{\text {pulse }}(\mathrm{s})\end{array}$ \\
\hline J0117.6 - 7330 & $\mathrm{TP}$ & $\begin{array}{l}011741.4 \\
-73 \quad 3049\end{array}$ & Be star & $\begin{array}{l}14.19,-0.07,-- \\
\text { B0.5IIIe, } 0.03\end{array}$ & 10 & 22.07 \\
\hline $\mathrm{C}, \mathrm{R}$ & $\mathrm{x} 0.6^{\prime \prime}$ & $300.4,-43.5$ & & {$[90,649]$} & [439] & [439] \\
\hline
\end{tabular}

Pos.: [255]; in SMC: [98]; X-ray outburst: [98]; IR excess and strong $\mathrm{H} \alpha$ emission: [90, 112]; projected rotational velosity $145 \mathrm{~km} \mathrm{~s}^{-1}$ : [649]; mass of the Be star $18 M_{\odot}$ : [649]; opt. phot. \& spectroscopy: [649]; soft X-ray spectrum: [98]; hard X-ray spectrum: [439]; black hole candidate: [98].

\begin{tabular}{|c|c|c|c|c|c|c|}
\hline $\mathrm{J} 0146.9+6121$ & & 014332.6 & $\mathrm{LS} \mathrm{I}+61^{\circ} 235$ & $11.33,0.82,-0.39$ & $1-3$ & \\
\hline $\mathrm{R}, \mathrm{AS}, \mathrm{X}, \mathrm{B}$ & o $2^{\prime \prime}$ & $\begin{array}{l}+610626 \\
129.2,-1.2\end{array}$ & [267] & $\begin{array}{l}\mathrm{B} 1 \mathrm{Ve}, 0.87 \\
{[143,410,579,640]}\end{array}$ & $\begin{array}{l}(0.1-2.4 \mathrm{keV}) \\
{[499]}\end{array}$ & $\begin{array}{l}1404.2 \\
{[254]}\end{array}$ \\
\hline
\end{tabular}

Pos.: [267]; in open cluster NGC 663: [438]; $v_{\mathrm{r}} \sin i \sim 250 \mathrm{~km} \mathrm{~s}^{-1}$ : [640]; X-ray obs.: [480]; new X-ray outburst: [254]; physical parameters and $V / R$ veriability: [579].

\begin{tabular}{|c|c|c|c|c|c|c|}
\hline $0236+610$ & & 023640.6 & $\mathrm{LS} \mathrm{I}+61^{\circ} 303$ & $10.7,0.8,-0.3$ & 0.2 & 26.45 \\
\hline & & +610054 & V615 Cas & B0e, 0.75 & $(0.2-5 \mathrm{keV})$ & \\
\hline $\mathrm{E}$ & $\mathrm{o}, \mathrm{r}$ & $135.7,+1.1$ & {$[70,267]$} & {$[180,308,600]$} & {$[48]$} & [677] \\
\hline
\end{tabular}

Pos.: [242]; X-ray source: [48]; radio outbursts: [677, 678]; four-year modulation radio outbursts: [185, 243, 534]; VLBI radio jet: [467, 679]; optical light curve: [472, 532, 533]; opt. spectra: [5, 308]; orbital solution: [308, 463]; $v_{\mathrm{r}} \sin i \sim 200 \mathrm{~km} \mathrm{~s}{ }^{-1}$ : [144]; system velocity: [721]; long-term optical obs.: [242, 423]; IR obs.: [157, 303]; distance: [216, 653]; UV obs.: [294, 307, $308,445]$; wind structure: [746]; related to $\gamma$ source CG 135+1(?): [240, 276]; simultaneous X-ray/radio obs.: [268]; black hole candidate: [567]; long-term radio obs.: [572]; multiwavelength obs.: [652]; orbital period detected in photometry, X-ray and $\mathrm{H} \alpha$ emission line: [535, 536, 791].

\begin{tabular}{lllllll}
\hline $0331+530$ & TP & 033114.9 & BQ Cam & $15.1-15.4,1.6-2.3,--<0.5-1250$ & 34.25 \\
& & +530024 & O8.5Ve, 1.9 & & 4.4 & \\
V, T, Exo & o & $146.1,-2.2$ & {$[381]$} & {$[128,290,513]$} & {$[655,683]$} & {$[508,655,771]$}
\end{tabular}

Pos.: [381]; X-ray outbursts: [655, 683, 771]; X-ray obs.: [444]; rapid X-ray variability: [38]; X-ray cyclotron line: [443]; X-ray pulse phase sp.: [705]; optical id.: [42, 381]; optical spectrum: [661]; $v_{\mathrm{r}} \sin i \sim 150 \mathrm{~km} \mathrm{~s}^{-1}$ : [128]; system velocity: [721]; H $\alpha$ emission: [338]; IR obs.: [106]; wind structure: [746]; tilt between orbital and equatorial planes: [514]; QPO: [669].

\begin{tabular}{lllllll}
\hline $0352+309$ & $\mathrm{P}$ & 035215.1 & $\mathrm{X}$ Per & $6.0-6.6,0.29,-0.82$ & $<9-37$ & $580 ?$ \\
& & +305401 & & B0 III-Ve, 0.4 & 835 \\
$\mathrm{U}, \mathrm{M}, \mathrm{A}, \mathrm{H}, \mathrm{S}$ & $\mathrm{o}$ & $163.1,-17.1$ & {$[64]$} & {$[311,434,489,639]$} & {$[63]$} & {$[306,508]$}
\end{tabular}

Pos.: [63]; X-rays: [591, 754]; X-ray sp.: [765]; optical id.: [71]; long-term optical record: [298, 422, 494]; reported 580 day period unlikely to be orbital: [306, 311, 548]; disappearance emission lines: [520]; system velocity: [721]; $v_{\mathrm{r}} \sin i \sim$ $250-400 \mathrm{~km} \mathrm{~s}^{-1}$ : [306, 489, 761]; UV obs.: [43, 261]; stellar parameters: [190]; comparison with $\gamma$ Cas: [489, 764]; opt. polarimetry: [396]; envelope structure: [682]; photometry and polarimetry obs. during disc loss: [595]; fundamental parameters: [434]; multiwavelength study: [594]; orbital period of 250.3 d: [Delgado-Marti H., et al., 2000, ApJ (submitted)].

\begin{tabular}{|c|c|c|c|c|c|}
\hline J0421+560 & $\mathrm{T}$ & $\begin{array}{l}041946.0 \\
555924\end{array}$ & CI Cam & $\begin{array}{l}9.25,1.0,-0.4 \\
--, 0.8\end{array}$ & 2000 \\
\hline $\mathrm{X}, \mathrm{B}, \mathrm{C}, \mathrm{AS}$ & x $50^{\prime \prime}$ & $149.2,+4.1$ & & {$[100 \mathrm{a}, 323]$} & {$[40,523]$} \\
\hline
\end{tabular}

Pos.: [523]; X-ray outbursts: [643]; multi-wavelength obs.: [40]; optical outbursts: [593]; radio obs.: [287]; radio position: [285]; likely a black hole candidate: [40]; B[e]: [40]; an unusual symbiotic-type X-ray binary (?): [220]; distance $\geq 2$ kpc: [40]; sgB[e] star: [100]; IR spe.: [100]; opt./IR/radio obs.: [100a]; long-term UBVRIJHK obs.: [41a]; X-ray/opt. obs.: [219a].

\begin{tabular}{lllllll}
\hline J0440.9+4431 & $\mathrm{P}$ & 044059.9 & BSD 24-491 & $10.78,0.61,-0.36$ & 1 & \\
& & 443151 & & B0V-IIIe & & 202.5 \\
$\mathrm{R}, \mathrm{X}$ & $\mathrm{x} 17^{\prime \prime}$ & $159.8,-1.3$ & {$[142]$} & {$[500]$} & {$[577]$} & {$[577]$}
\end{tabular}

Pos.: [500]; distance 3.2 kpc: [500]; optical spe.: [500]. 
Table 1. continued

\begin{tabular}{|c|c|c|c|c|c|c|}
\hline Name(s) & $\begin{array}{l}\text { type } \\
\text { Pos. }\end{array}$ & $\begin{array}{l}\mathrm{RA} \\
\mathrm{DEC} \\
l^{\mathrm{II}}, b^{\mathrm{II}} \\
\end{array}$ & Opt. Ctp. & $\begin{array}{l}V, B-V, U-B \\
\text { Sp. type, } E_{B-V}\end{array}$ & $\begin{array}{l}F_{x} \\
\mu \mathrm{Jy}\end{array}$ & $\begin{array}{l}P_{\text {orb }}(\mathrm{d}) \\
P_{\text {pulse }}(\mathrm{s})\end{array}$ \\
\hline J0501.6 - 7034 & & 050123.9 & Be star & $14.5,-0.07,--$ & & \\
\hline CAL 9 & & -703333 & & $\mathrm{~B} 0 \mathrm{e},-$ - & & \\
\hline $\mathrm{E}, \mathrm{R}$ & x $2.9^{\prime \prime}$ & $281.9,-34.5$ & {$[618]$} & {$[618]$} & & \\
\hline \multicolumn{7}{|c|}{ Pos.: [612]; in LMC: [618]; opt. cpt. HV 2289: [618]; IUE ultraviolet obs.: [620]. } \\
\hline J0502.9-6626 & $\mathrm{TP}$ & 050251.6 & Be star & $14.22,-0.10,--$ & 5.7 & \\
\hline CAL E & & -662625 & & $\mathrm{~B} 0 \mathrm{e},--$ & & 4.0635 \\
\hline $\mathrm{E}, \mathrm{R}$ & $\mathrm{x} 1.2^{\prime \prime}$ & $277.0,-35.4$ & {$[618]$} & {$[617]$} & [619] & [619] \\
\hline
\end{tabular}

Pos.: [612]; in LMC: [618]; highly variable X-ray emission: [618]; IUE ultraviolet obs.: [620].

\begin{tabular}{ll}
\hline J0512.6-6717 & 051241.8 \\
& -671723 \\
$\mathrm{R}$ & $\mathrm{x} 40^{\prime \prime} \quad 277.8,-34.3$
\end{tabular}

Pos.: [250]; a HMXB in LMC (?): [250]; weak source, hard X-ray spectrum: [250].

\begin{tabular}{llllll}
\hline J0516.0-6916 & $\mathrm{T}$ & 051600.1 & star 2 & $15.0,-0.1,-0.9$ & 0.07 \\
& & -691609 & & B1e & \\
$\mathrm{R}$ & $\mathrm{x} 7.3^{\prime \prime}$ & $280.1,-33.6$ & {$[141]$} & {$[141]$} & {$[141]$}
\end{tabular}

Pos.: [612]; LMC member: [141].

\begin{tabular}{|c|c|c|c|c|}
\hline J0520.5 - 6932 & $\mathrm{~T}$ & $\begin{array}{l}052030.3 \\
-693204\end{array}$ & Be star & $\begin{array}{l}14.4,--,-- \\
\text { O8e, - - }\end{array}$ \\
\hline $\mathrm{R}$ & $\mathrm{x} 16.6^{\prime \prime}$ & $280.3,-33.2$ & [618] & {$[618]$} \\
\hline
\end{tabular}

Pos.: [250]; in LMC: [618]; IUE ultraviolet obs.: [620]; not detected with Einstein: [618].

\begin{tabular}{|c|c|c|c|c|c|}
\hline \multirow{3}{*}{$\begin{array}{l}0521+373 \\
\mathrm{U}, \mathrm{H}\end{array}$} & & 051910.7 & HD 34921 & $7.51,0.14,-0.86$ & 1 \\
\hline & & +373744 & SAO57950 & B0 IVpe, 0.42 & \\
\hline & o & $170.0,+0.7$ & {$[646]$} & {$[78,555,599]$} & {$[215,781]$} \\
\hline
\end{tabular}

Pos.: [646]; hard X-ray spectrum: [555]; UV/opt./IR obs.: [555, 599]; IR spe.: [100]; unclB[e] star: [100].

\begin{tabular}{lllllll}
\hline J0529.8-6556 & TP & 052948.4 & GSC 8891.0213 & $B=14.5, R=13.3$ & 0.17 & \\
& & -655651 & & B2III-Ve, - - & 69.5 \\
$\mathrm{R}$ & $\mathrm{x} 8^{\prime \prime}$ & $275.9,-32.9$ & {$[253]$} & {$[253]$} & {$[253]$} & {$[253]$}
\end{tabular}

Pos.: [253]; LMC member: [253]; opt. spectrum: [253].

\begin{tabular}{|c|c|c|c|c|c|}
\hline \multirow[t]{2}{*}{$053109-6609.2$} & TP & 053109 & & 1 & 25.4 \\
\hline & & -660912 & $\mathrm{Be} ?, 0.1$ & & 13.7 \\
\hline Exo, SL, B, R & $\mathrm{x} 10^{\prime \prime}$ & $276.2,-32.7$ & {$[265]$} & {$[265]$} & [165] \\
\hline
\end{tabular}

Pos.: [530]; in LMC: [265]; hard X-ray spectrum: [265]; X-ray outburst: [75]; not detected with Einstein: [265]; optical cpt. probably Be star: [265]; orbital period between 4 and 40 days, mostly 25.4 days: [165].

\begin{tabular}{llll}
\hline J0531.5 - 6518 & 053136.1 & \\
& & -651816 \\
& & \\
$\mathrm{R}$ & $\mathrm{x} \mathrm{18.8^{ \prime \prime }}$ & $275.1,-32.7$ & {$[250]$}
\end{tabular}

Pos.: [250]; HMXB in LMC: [250]. 
Table 1. continued

\begin{tabular}{|c|c|c|c|c|c|c|}
\hline Name(s) & $\begin{array}{l}\text { type } \\
\text { Pos. }\end{array}$ & $\begin{array}{l}\mathrm{RA} \\
\mathrm{DEC} \\
l^{\mathrm{II}}, b^{\mathrm{II}}\end{array}$ & Opt. Ctp. & $\begin{array}{l}V, B-V, U-B \\
\text { Sp. type, } E_{B-V}\end{array}$ & $\begin{array}{l}F_{x} \\
\mu \mathrm{Jy}\end{array}$ & $\begin{array}{l}P_{\text {orb }}(\mathrm{d}) \\
P_{\text {pulse }}(\mathrm{s})\end{array}$ \\
\hline $0532-664$ & $\mathrm{P}$ & 053247.3 & $\mathrm{Sk}-\mathrm{Ph}$ & $14.0,-0.1,-1.1$ & $<3-60$ & 1.40 \\
\hline $\begin{array}{l}\mathrm{LMC} \mathrm{X}-4 \\
\mathrm{U}, \mathrm{M}, \mathrm{A}, \mathrm{H}, \mathrm{AS}, \\
\text { G. R }\end{array}$ & o $2^{\prime \prime}$ & $\begin{array}{l}-662413 \\
276.3,-32.5\end{array}$ & {$[93]$} & $\begin{array}{l}\text { O7 III-V, } 0.1 \\
{[280,365,431]}\end{array}$ & {$[63]$} & $\begin{array}{l}13.5 \\
{[365,508]}\end{array}$ \\
\hline
\end{tabular}

Pos.: [63]; X-ray orbital parameters: [365, 411, 553]; X-ray eclipses: [413, 756]; 30 day period X-ray: [400]; X-ray spectrum: [665, 765]; rapid X-ray variability: [38]; X-ray flare: [553]; opt. light curve: [92, 271, 326]; opt. spectrum \& radial velocity curve: [314]; $v_{\mathrm{r}} \sin i \sim 170 \mathrm{~km} \mathrm{~s}^{-1}$ : [314]; opt. 30 day period: [271, 326]; precessing-disk model: [271]; UV obs.: [56, 714]; UV/X-ray obs.: [738]; orbital decay: [607, 780].

\begin{tabular}{|c|c|c|c|c|c|}
\hline \multirow[t]{2}{*}{ J0532.4-6535 } & & 053225.3 & RGC 36 & $\begin{array}{l}J=11.9, H=11.1 \\
K=10.7\end{array}$ & 0.01 \\
\hline & $\mathrm{x} 174^{\prime \prime}$ & -653509 & & {$[575]$} & $(0.1-2.4 \mathrm{keV})$ \\
\hline
\end{tabular}

Pos.: [250]; hard X-ray spectrum: [250]; Be star in LMC: [250].

\begin{tabular}{llllll}
\hline J0532.5-6551 & $\mathrm{V}$ & 053232.6 & Sk-65 66 & $12.3,-,--$ & 0.03 \\
& & -655140.8 & & OB & $(0.1-2.4 \mathrm{keV})$ \\
$\mathrm{R}$ & $\mathrm{o}$ & $275.7,-32.6$ & {$[609]$} & {$[609]$} & {$[253]$}
\end{tabular}

Pos.: [609]; in LMC: [253]; long-term X-ray variation [253]; Black hole candidate or a NS: [253].

\begin{tabular}{lllllll}
\hline J0535.0-6700 & $\mathrm{T}$ & 053505.9 & RGC 28 & $18.1,--,--$ & 0.04 & $241 ?$ \\
& & -670016 & & Be?,- - & $(0.1-2.4 \mathrm{keV})$ & \\
$\mathrm{R}$ & $\mathrm{x} \mathrm{4} 4^{\prime \prime}$ & $277.1,-32.2$ & {$[575]$} & {$[575]$} & {$[250]$} & {$[575]$}
\end{tabular}

Pos.: [250]; in LMC: [250]; IR photometry: [575]; Be/X-ray system: [250].

\begin{tabular}{lllllll}
\hline $0535-668$ & PT & 053542.4 & ${ }^{*} \mathrm{Q}$ & $12.3-14.9,0.1,-0.9$ & $<0.01-180$ & 16.7 \\
$0538-66$ & & -665339 & & B2 III-Ve, 0.1 & 0.069 \\
$\mathrm{~A}$ & o $2^{\prime \prime}$ & $276.9,-32.2$ & {$[89,350]$} & {$[89,167,431,528]$} & {$[63]$} & {$[508,636]$}
\end{tabular}

Pos.: [350]; in LMC: [348, 528]; X-ray outbursts: [348, 757]; X-ray pulsations: [637]; optical outbursts: [89, 126, 167, 636, 729]; long-term off states: [430, 528]; quiescent opt. phot. \& spectroscopy: [126, 320, 641, 642, 709, 729]; very strong emission lines during outbursts: [89]; IR obs.: [8]; opt. polarimetry: [102]; UV obs.: [89, 295, 573]; wind structure: [746]; X-ray obs. during quiescence: [484]; accretion to magnetosph.: [130].

\begin{tabular}{|c|c|c|c|c|c|c|}
\hline $0535+262$ & $\mathrm{TP}$ & 053548.0 & V725 Tau & $\begin{array}{l}8.9-9.6,0.45-0.62 \\
-0.54\end{array}$ & $<3-2800$ & 111 \\
\hline $\mathrm{U}, \mathrm{A}, \mathrm{H}$ & o & $\begin{array}{l}+261718 \\
181.4,-2.6\end{array}$ & $\begin{array}{l}\text { HD } 245770 \\
{[414]}\end{array}$ & $\begin{array}{l}\text { O9.7IIIe, } 0.8 \\
{[225,449,653,660]}\end{array}$ & [63] & $\begin{array}{l}104 \\
{[508,561]}\end{array}$ \\
\hline
\end{tabular}

Pos.: [63]; transient: [509, 598]; long-term X-ray obs.: [561]; hard X-ray obs.: [108, 371, 585, 628]; $55.7 d$ orbit?: [754]; longterm optical record: [41, 660]; opt. phot.: [266, 634]; opt. spectr.: [4, 313, 319]; $v_{\mathrm{r}} \sin i \sim 300 \mathrm{~km} \mathrm{~s}^{-1}$ : [313]; system velocity: [721]; multi-wavelength obs.: [164, 341]; X-ray/opt. relation: [436]; UV obs.: [164, 546, 784]; accretion model: [498]; wind structure: [746]; 1992 review: [230]; disc loss \& renewal: [257]; IR spe. obs.: [99a]; distance: [653]; long-term opt./UV spe.: [99]; cyclotron line: [20, 372]; QPO during a giant outburst: [208].

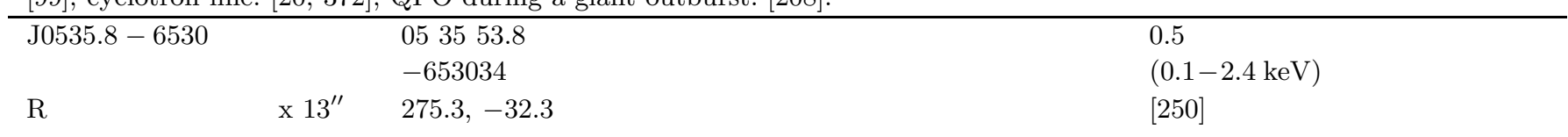

Pos.: [250]; likely a HMXB in LMC: [250].

\begin{tabular}{|c|c|c|c|c|c|c|}
\hline $\begin{array}{l}0538-641 \\
\text { LMC X-3 }\end{array}$ & $\mathrm{U}$ & $\begin{array}{l}053839.7 \\
-640634\end{array}$ & ${ }^{*} 1$ & $\begin{array}{l}16.7-17.5,-0.2,-0.6 \\
\text { B3 Ve, } 0.1\end{array}$ & $<1.7-44$ & 1.70 \\
\hline $\mathrm{U}, \mathrm{M}, \mathrm{A}, \mathrm{H}, \mathrm{F}, \mathrm{X}$ & o $3^{\prime \prime}$ & $273.6,-32.1$ & {$[716]$} & {$[136,431,708]$} & [63] & {$[136]$} \\
\hline
\end{tabular}

Pos.: [38]; X-ray obs.: [349]; long-term X-ray obs.: [545]; rapid X-ray variability: [38]; X-ray spectrum: [665, 693, 759, 766]; 199 d X-ray period: [138, 665]; opt. lt crv.: [50, 730]; mass function: [136, 395, 525]; $v_{\mathrm{r}} \sin i \sim 130 \mathrm{~km} \mathrm{~s}^{-1}$ : [136]; UV obs.: [139, 694, 695]; X-ray/UV/opt. obs.: [695]; near IR sp.: [137]. 
Table 1. continued

\begin{tabular}{|c|c|c|c|c|c|c|}
\hline Name(s) & $\begin{array}{l}\text { type } \\
\text { Pos. }\end{array}$ & $\begin{array}{l}\mathrm{RA} \\
\mathrm{DEC} \\
l^{\mathrm{II}}, b^{\mathrm{II}}\end{array}$ & Opt. Ctp. & $\begin{array}{l}V, B-V, U-B \\
\text { Sp. type, } E_{B-V}\end{array}$ & $\begin{array}{l}F_{x} \\
\mu \mathrm{Jy}\end{array}$ & $\begin{array}{l}P_{\text {orb }}(\mathrm{d}) \\
P_{\text {pulse }}(\mathrm{s})\end{array}$ \\
\hline $0540-697$ & $\overline{\mathrm{U}}$ & 054005.5 & ${ }^{*} 32$ & $14.5,0.29,-0.70$ & $3-25$ & 4.22 \\
\hline LMC X-1 & & -694604 & & O7-9 III, 0.37 & & \\
\hline $\mathrm{U}, \mathrm{M}, \mathrm{A}, \mathrm{H}, \mathrm{X}$ & o $3^{\prime \prime}$ & $280.2,-31.5$ & {$[135,181]$} & {$[47,318]$} & [63] & {$[322]$} \\
\hline
\end{tabular}

Pos.: [63]; ultra-soft X-ray spe.: [759]; X-ray spe. \& QPO: [184]; rapid X-ray variability: [38]; X-ray obs.: [349, 665]; mass function: [322]; $v_{\mathrm{r}} \sin i \sim 150 \mathrm{~km} \mathrm{~s}^{-1}$ : [318]; UV obs.: [47, 322]; in He III region: [527]; opt. cpt. uncertainty (O7III or supergiant R148): [140].

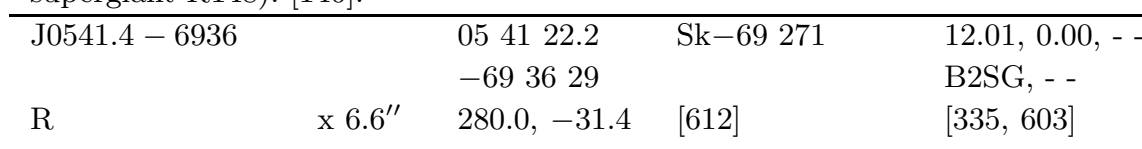

Pos.: [612]; supergiant in LMC: [612].

\begin{tabular}{llll}
\hline J0541.5-6833 & 054137.1 & LMC BI267 & $14.02,-0.03,-1.04$ \\
& -683232 & OB0 \\
R & x 4.5" & $278.8,-31.4$ & {$[74]$}
\end{tabular}

Pos.: [74]; LMC member: [612].

\begin{tabular}{|c|c|c|c|c|c|}
\hline $0544-665$ & & $\begin{array}{l}054415.6 \\
-663459\end{array}$ & $* 1$ & $\begin{array}{l}15.4,-0.20,-0.96 \\
\text { B1 Ve, } 0.1\end{array}$ & 1.8 \\
\hline $\mathrm{H}$ & o $3^{\prime \prime}$ & $276.5,-31.4$ & [349] & {$[431,715]$} & [349] \\
\hline
\end{tabular}

Pos.: [38]; optical id. not completly certain: [715]; *1 member of LMC: [715].

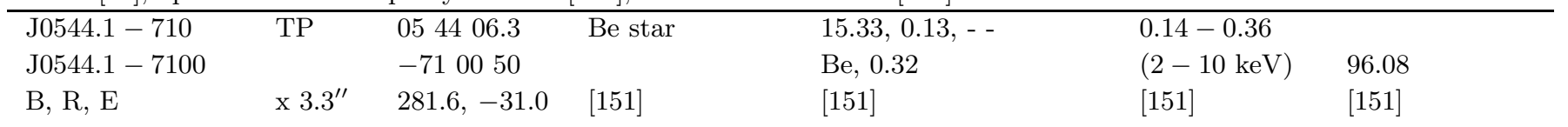

Pos.: [250]; in LMC: [151]; very hard X-ray spectrum: [151]; pronounced H $\alpha$ activity from a likely Be-type star: [151].

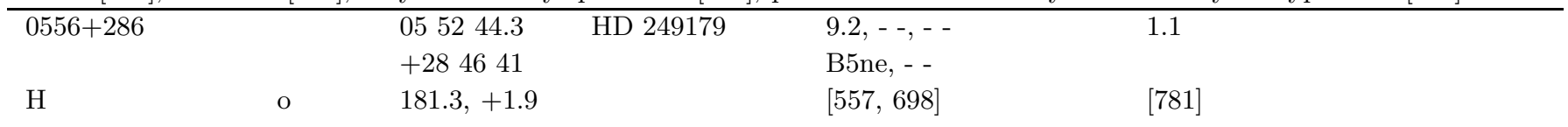

Pos.: [698]; Be star: [739].

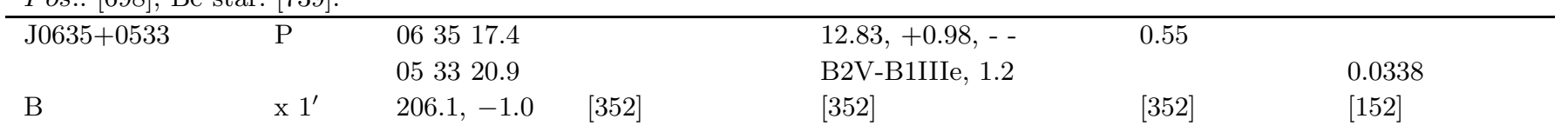

Pos.: [352]; opt. emission line: [352]; within the error box of the GeV $\gamma$-ray source 2EG J0635+0521: [352]; distance $\sim 2.5-5 \mathrm{kpc}:[352]$.

\begin{tabular}{lllllll}
\hline J0648.0-4419 & $\mathrm{P}$ & 064804.6 & HD 49798 & $8.27,-0.24,-1.18$ & 0.03 & 1.55 \\
& & -441854.4 & & sdO6, - & 13.1789 \\
R, B & $\mathrm{x} 10^{\prime \prime}$ & $253.7,-19.1$ & {$[329]$} & {$[344]$} & {$[329,684]$}
\end{tabular}

Pos.: [768]; distance 650 pc: [329]; very soft X-ray spe.: [329]; ultraviolet spe.: [73]; likely a NS: [329]; compact being a $\mathrm{WD}(?):[49]$.

\begin{tabular}{lllllll}
\hline $0726-260$ & TP & 072650.0 & LS 437 & $11.6,0.3,-0.6$ & $1.2-4.7$ & 34.5 \\
& & -260013 & & O8-9Ve, 0.6 & 103.2 \\
U, A, E, H, R, X & о & $240.3,-4.1$ & {$[654]$} & {$[515,654]$} & {$[743,781]$} & {$[131]$}
\end{tabular}

Pos.: [654]; distance 4.6 or 6.1 kpc: [124a, 515]; X-ray flare: [654]; opt./IR obs.: [515]; orbital and spin periods: [131]. 
Table 1. continued

\begin{tabular}{|c|c|c|c|c|c|c|}
\hline Name(s) & $\begin{array}{l}\text { type } \\
\text { Pos. }\end{array}$ & $\begin{array}{l}\mathrm{RA} \\
\mathrm{DEC} \\
l^{\mathrm{II}}, b^{\mathrm{II}}\end{array}$ & Opt. Ctp. & $\begin{array}{l}V, B-V, U-B \\
\text { Sp. type, } E_{B-V}\end{array}$ & $\begin{array}{l}F_{x} \\
\mu \mathrm{Jy}\end{array}$ & $\begin{array}{l}P_{\text {orb }}(\mathrm{d}) \\
P_{\text {pulse }}(\mathrm{s})\end{array}$ \\
\hline $0739-529$ & $\mathrm{O}$ & $\begin{array}{l}074609.8 \\
-5312 \quad 28 \\
266.4,-13.7\end{array}$ & $\begin{array}{l}\text { HD } 63666 \\
\text { SAO235515 } \\
{[646]}\end{array}$ & $\begin{array}{l}7.62,0.02,-0.24 \\
\text { B7 IV-Ve, - - } \\
{[293,437,518,698]}\end{array}$ & {$[781]$} & \\
\hline Pos.: [698]. & & & & & & \\
\hline $0749-600$ & o & $\begin{array}{l}075527.7 \\
-605754 \\
274.0,-16.2\end{array}$ & $\begin{array}{l}\text { HD } 65663 \\
\text { SAO250018 } \\
{[646]}\end{array}$ & $\begin{array}{l}6.73,0.05,-0.25 \\
\text { B8 IIIe, } 0.09 \\
{[153,154,698]}\end{array}$ & 0.7 & \\
\hline
\end{tabular}

Pos.: [698]; Be star: [739]; in open cluster NGC 2516: [153].

\begin{tabular}{|c|c|c|c|c|c|c|}
\hline J0812.4-3114 & x $21^{\prime \prime}$ & $\begin{array}{l}081228.4 \\
-311451 \\
249.6,+1.6\end{array}$ & LS 992 & $\begin{array}{l}12.42,0.41,-0.69 \\
\text { B0.5V-IIIe, - - } \\
{[500]}\end{array}$ & [500] & $\begin{array}{l}80 \\
31.8851 \\
{[125,577]}\end{array}$ \\
\hline \multicolumn{7}{|c|}{ Pos.: [500]; distance 9.2 kpc: [500]; optical spectrum: [500]. } \\
\hline $0834-430$ & $\mathrm{TP}$ & $\begin{array}{l}083410 \\
-4300.6\end{array}$ & $\operatorname{star}^{*} \mathrm{D}$ & $\begin{array}{l}20.4,--,-- \\
\text { B0-2 III-Ve, } 4.0\end{array}$ & $30-300$ & $\begin{array}{l}110 \\
12.3\end{array}$ \\
\hline $\mathrm{Gr}, \mathrm{R}, \mathrm{K}, \mathrm{G}, \mathrm{C}$ & $\mathrm{x} 1^{\prime}$ & $262.0,-1.5$ & {$[334 \mathrm{a}]$} & {$[334 \mathrm{a}]$} & {$[239]$} & {$[239,773]$} \\
\hline
\end{tabular}

Pos.: [269]; more accurate pos. for epoch 2000: 0835 55.4, -43 1111.9 (o $1^{\prime \prime}$ ): [334a]; sequence X-ray outbursts: [774]; eccentricity $0.1<e<0.17$ : [774]; strong H $\alpha$ emission: [334a]; southern of two neighboring sources: [239, 440]; recurrent transient: [401]; distance $3-5 \mathrm{kpc:} \mathrm{[334a];} \mathrm{not} \mathrm{the} \mathrm{same} \mathrm{transient} \mathrm{as} \mathrm{MX} 0836$ - 42: [115, 453]; X-ray obs.: [16]; X-ray/opt. obs.: [39]; IR/opt obs.: [334a].

\begin{tabular}{lllllll}
\hline $0900-403$ & $\mathrm{P}$ & 090013.2 & HD 77581 & $6.9,0.47,-0.51$ & $2-1100$ & 8.96 \\
Vela X-1 & & -402125 & GP Vel & B0.5 Ib, 0.7 & & 283 \\
U, M, A, H, B, X, & o & $263.1,+3.9$ & {$[213]$} & {$[183,495,518,717]$} & {$[63]$} & {$[162,508,711]$}
\end{tabular}

$\mathrm{AS}, \mathrm{R}$

Pos.: [63]; X-ray sp.: [510, 891, 614, 765]; cyclotron line: [371]; pulse timing \& X-ray orbit: [61, 62, 161, 162, 571, 696, 711]; X-ray eclipses: [213, 703]; limits on apsidal motion: [160]; rapid X-ray variability: [38]; orbital variation X-ray absorption: [251]; energy dependence pulse profile: [570]; opt. light curve: [374, 689]; long-term cycles (?): [559, 689]; opt. radial-velocity curve: [720, 726]; system velocity: [721]; $v_{\mathrm{r}} \sin i \sim 90-130 \mathrm{~km} \mathrm{~s}^{-1}:[482,689,772]$; UV obs.: [183, 357, 470, 546, 606]; high-resolution IUE obs.: [659]; opt. polarimetry: [173]; IR obs.: [232]; bow shock around the sys. [361]; polarization: [779].

\begin{tabular}{lllllll}
\hline J1008-57 & TP & 100946 & star & $15.27,-1.66,--$ & 1200 & 135 \\
& & -581732 & & O9-B1, 1.9-2.0 & 93.5 \\
C, R, AS, Exo & x 15" & $283.0,-1.8$ & {$[109]$} & {$[109,587]$} & {$[663]$} & {$[550,635,663]$}
\end{tabular}

Pos.: [550]; IR and optical spectral obs.: [109]; 260 days orbital period: [206]; distance 5 kpc: [109]; large IR excess: [109].

\begin{tabular}{lllllll}
\hline $1024.0-5732$ & $\mathrm{P}$ & 102405.4 & Wack 2134 & $12.7,1.5,--$ & $1-10$ & \\
& & -573324 & TH $\alpha 35-42$ & O5:, 1.8 & $(0.2-4.5 \mathrm{keV})$ & 0.061 \\
$\mathrm{E}$ & o $6^{\prime \prime}$ & $284.5,-0.2$ & {$[686]$} & {$[81]$} & {$[81]$} & {$[81]$}
\end{tabular}

Pos.: [81]; no optical pulsations: [169]; emission line star: [739]; a Wolf-Rayet star+ O star sys. rather than a HMXB: [576].

\begin{tabular}{|c|c|c|c|c|c|}
\hline $1036-565$ & & 102828.3 & HD 91188 & $6.64,-0.10,-0.56$ & 3.3 \\
\hline $4 \mathrm{U} 1036-56$ & & -564914 & SAO238130 & B4 IIIe, - - & \\
\hline A & $\mathrm{O}$ & $284.6,+0.7$ & [646] & {$[221,518]$} & [743] \\
\hline
\end{tabular}

Pos.: [698]; probably the same source as RX J1037.5 - 5674: [500]; Be star: [739]; X-ray flare 1974 Nov.: [743]; periodic (2.924d) optical brightness variations reflect rotation of Be star: [27, 28]; IR spe.: [474]. 
Table 1. continued

\begin{tabular}{|c|c|c|c|c|c|c|}
\hline Name(s) & $\begin{array}{l}\text { type } \\
\text { Pos. }\end{array}$ & $\begin{array}{l}\mathrm{RA} \\
\mathrm{DEC} \\
l^{\mathrm{II}}, b^{\mathrm{II}}\end{array}$ & Opt. Ctp. & $\begin{array}{l}V, B-V, U-B \\
\text { Sp. type, } E_{B-V}\end{array}$ & $\begin{array}{l}F_{x} \\
\mu \mathrm{Jy}\end{array}$ & $\begin{array}{l}P_{\text {orb }}(\mathrm{d}) \\
P_{\text {pulse }}(\mathrm{s})\end{array}$ \\
\hline J1037.5 - 5647 & $\mathrm{P}$ & $\begin{array}{l}103735.2 \\
-564759\end{array}$ & LS 1698 & $\begin{array}{l}11.3,0.9,-- \\
\text { B0V-IIIe, } 0.75\end{array}$ & 0.17 & 862 \\
\hline $\mathrm{R}$ & $\mathrm{x} 21^{\prime \prime}$ & $285.4,+1.5$ & {$[500]$} & {$[500]$} & {$[577]$} & {$[577]$} \\
\hline
\end{tabular}

Pos.: [500]; distance 5.0 kpc: [500]; HD 91188 not the opt. cpt.: [500]; probably same source as 4U 1036 - 56: [500].

\begin{tabular}{llllll}
\hline $1048.1-5937$ & $\mathrm{P}$ & 104809.0 & $<0.1-1$ \\
$\mathrm{E}$ & $\mathrm{x} 9^{\prime \prime}$ & $289.2,-0.5$ & {$[477]$} & {$[632]$} & {$[124,632]$}
\end{tabular}

Pos.: [477]; discovery: [632]; Be/X or LMXB (?): [124]; opt. phm. and sp. of possible counterparts: [477]; excluding Mainsequence companion: [479].

\begin{tabular}{lllllll}
\hline $1118-615$ & PT & 111845.2 & He3-640 & $12.1,0.96,-0.30$ & $0.1-70$ & 405 \\
& & -613831 & & O9.5 III-Ve, 1.2 & & \\
A & o & $292.5,-0.9$ & {$[339]$} & {$[339]$} & {$[63,497]$}
\end{tabular}

Pos.: [63]; X-ray transient: [187, 336, 433]; opt. phot. \& spe.: [339]; sys. velocity: [721]; $v_{\mathrm{r}} \sin i \sim 300 \mathrm{~km} \mathrm{~s}^{-1}$ : [339]; X-ray/opt. obs.: [497]; opt. spe. after X-ray outburst: [556]; multiwavelength study: [109]; UV obs.: [105]; radio obs.: [297].

\begin{tabular}{lllllll}
\hline $1119-603$ & $\mathrm{P}$ & 111901.9 & V779 Cen & $13.3,1.07,-0.04$ & $10-312$ & 2.09 \\
Cen X-3 & & -602057 & & O6.5 II-III, 1.4 & & 4.84 \\
$\mathrm{U}, \mathrm{M}, \mathrm{A}, \mathrm{H}$ & ${\text { o } 3^{\prime \prime}}$ & $292.1,+0.3$ & {$[72,394]$} & {$[315,394,586]$} & {$[63]$} & {$[366,508]$}
\end{tabular}

Pos.: [63]; X-ray pulsations: [224, 696]; X-ray orbit: [366]; period decay: [188, 366]; X-ray spectrum: [25, 76, 432, 765]; long-term X-ray history: [246, 328, 558, 562, 624]; X-ray eclipses: [622]; X-ray obs.: [296, 504, 512]; aperiodic X-ray variability: [38]; QPO: [668]; opt. light curve: [373, 689, 727]; opt. radial-velocity curve: [315, 502, 524]; mass of the NS: [22]; $v_{\mathrm{r}} \sin i \sim 250 \mathrm{~km} \mathrm{~s}^{-1}:$ [315]; system velocity: [721]; IR obs.: [232]; structure companion star: [97]; distance: [302]; outburst of $\mathrm{GeV} \gamma$-ray emission: [733].

\begin{tabular}{lllllll}
\hline $1145-619$ & PT & 114533.6 & Hen 715 & $9.3,0.18,-0.81$ & $4-1000$ & 187.5 \\
& & -615544 & HD 102567 & B1 Vne, 0.35 & 292.4 \\
$\mathrm{U}, \mathrm{M}, \mathrm{A}, \mathrm{H}, \mathrm{S}$ & $\mathrm{o}$ & $295.6,-0.2$ & {$[64]$} & {$[195,518,746]$} & {$[63]$} & {$[508,748]$}
\end{tabular}

Pos.: [63]; X-ray spectrum: [765]; X-ray obs.: [475]; long-term X-ray history: [562, 748]; opt. spectrum: [340, 762]; $v_{\mathrm{r}} \sin i \sim 270 \mathrm{~km} \mathrm{~s}^{-1}:$ [261, 340]; system velocity: [721]; coordinated X-ray/opt. obs.: [121]; UV obs.: [46, 163, 261, 546]; wind structure: [746]; multiwavelength obs.: [656].

\begin{tabular}{lllllll}
\hline $1145.1-6141$ & $\mathrm{P}$ & 114502.3 & V830 Cen & $13.1,1.5,0.15$ & $4-40$ & 5.65 \\
& & -614033 & & B2Iae, 1.6 & 298 \\
A, E, C & o $2^{\prime \prime}$ & $295.5,-0.0$ & {$[316]$} & {$[166,325]$} & {$[316]$} & {$[325,508]$}
\end{tabular}

Pos.: [316]; X-ray pulsations: [762]; orbital periods 10.76 d from opt. sp.: [321]; system velocity: [721]; 15' away from $1145-619:[397,763]$.

\begin{tabular}{lllllll}
\hline $1223-624$ & PT & 122349.7 & BP Cru & $10.8,1.76,0.42$ & $9-1000$ & 41.59 \\
GX 301-2 & & -622937 & Wra 977 & B1-1.5 Ia, 1.8 & 696 \\
U, M, A, H, S, o 3" & $300.1,-0.0$ & {$[64]$} & {$[59,260,688,734]$} & {$[63]$} & {$[508,613]$} \\
AS, C & & & &
\end{tabular}

$\mathrm{AS}, \mathrm{C}$

Pos.: [63]; X-ray spectrum: [765]; long-term X-ray record (recurrent outbursts near periastron): [560, 601, 749]; X-ray obs.: [248, 405, 588, 589, 760]; pulse-phase spectr.: [403]; pulse profile: [485]; X-ray orbit: [613]; X-ray dips: [404]; aperiodic X-ray variability: [38, 675]; rapid spin-up episodes: [383]; optical spectrum: [58, 260, 317, 539, 688]; optical photometry: [526, 723]; system velocity: [721]; IR obs.: [232]; a hypergiant (?): [360].

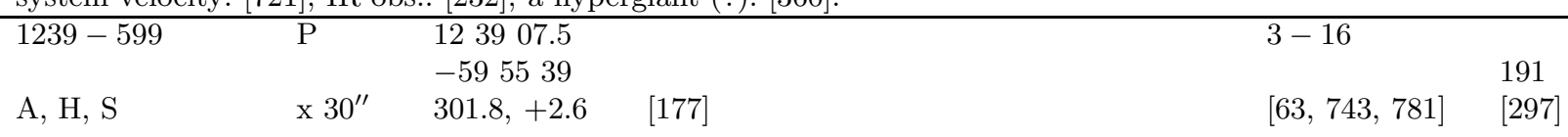


Table 1. continued

\begin{tabular}{|c|c|c|c|c|c|c|}
\hline Name(s) & $\begin{array}{l}\text { type } \\
\text { Pos. }\end{array}$ & $\begin{array}{l}\mathrm{RA} \\
\mathrm{DEC} \\
l^{\mathrm{II}}, b^{\mathrm{II}}\end{array}$ & $\begin{array}{l}\text { Opt. Ctp. } \\
{[\mathrm{FC}]}\end{array}$ & $\begin{array}{l}V, B-V, U-B \\
\text { Sp. type, } E_{B-V}\end{array}$ & $\begin{array}{l}F_{x} \\
\mu \mathrm{Jy}\end{array}$ & $\begin{array}{l}P_{\text {orb }}(\mathrm{d}) \\
P_{\text {pulse }}(\mathrm{s})\end{array}$ \\
\hline $1244-604$ & $\mathrm{~T}$ & $\begin{array}{l}124438 \\
-6022.2\end{array}$ & & & $<24-100$ & \\
\hline A & x $6.2^{\prime}$ & $302.5,+2.2$ & & & {$[82]$} & \\
\hline \multicolumn{7}{|l|}{ Pos.: [82]. } \\
\hline $1246-588$ & $\mathrm{~T}$ & $\begin{array}{l}124639 \\
-5851.0\end{array}$ & & & $<24-300$ & \\
\hline $\mathrm{U}, \mathrm{A}, \mathrm{H}$ & $\times 4.5^{\prime}$ & $302.7,+3.8$ & & & {$[82]$} & \\
\hline \multicolumn{7}{|l|}{ Pos.: [82]. } \\
\hline $1249-637$ & & $\begin{array}{l}123953.2 \\
-624706\end{array}$ & $\begin{array}{l}\text { HD } 110432 \\
\text { SAO252002 }\end{array}$ & $\begin{array}{l}5.31,0.27,-0.79 \\
\text { B0 IIIe, } 0.40\end{array}$ & 2.2 & \\
\hline $\mathrm{H}$ & o & $302.0,-0.2$ & {$[646]$} & {$[103,518]$} & {$[781]$} & \\
\hline
\end{tabular}

Pos.: [698]; Be star: [739]; UV obs.: [103]; interstellar abs. lines: [149]; optical obs.: [156, 196, 212]; variable radial velocity: [77, 685]; white-dwarf accretor(?): [745].

\begin{tabular}{|c|c|c|c|c|c|}
\hline $1253-761$ & & $\begin{array}{l}123559.8 \\
-750543\end{array}$ & $\begin{array}{l}\text { HD } 109857 \\
\text { SAO256967 }\end{array}$ & $\begin{array}{l}6.49,0.08,-0.24 \\
\text { B7 Vne, } 0.20\end{array}$ & 0.6 \\
\hline $\mathrm{H}$ & o & $302.1,-12.5$ & {$[646]$} & {$[103,518]$} & {$[781]$} \\
\hline
\end{tabular}

Pos.: [698]; Be star: [739]; visual double (sep. 2.2" $)$ : [201]; no optical brightness variations: [28]; white-dwarf accretor(?): [745].

\begin{tabular}{lllll}
\hline $1255-567$ & 125139.6 & $\mu^{2} \mathrm{Cru}$ & $5.17,-0.12,-0.51$ & 0.8 \\
& -565350 & HD 1120912 & B5 Ve, 0.04 & \\
$\mathrm{H}$ & $\mathrm{o}$ & $303.4,+5.7$ & & {$[518,639]$}
\end{tabular}

Pos.: [698]; Be star: [739]; $v_{\mathrm{r}} \sin i \sim 220 \mathrm{~km} \mathrm{~s}^{-1}$ : [639]; visual double with $\mu^{1}$ Cru: [288]; slow \& small optical variability: [549].

$\begin{array}{lllllll}\text { 1258-613 } & \text { PT? } & 125811.8 & \text { V 850 Cen } & 13.5-14.2,1.7,0.8 & 0.3-200 & 133 ? \\ \text { GX 304-1 } & & -611958 & { }^{*} 2(\mathrm{MMV}) & \text { B2 Vne, 2.0 } & & 272 \\ \text { U, M, A, S } & \text { o 2" } & 302.1,+1.2 & {[465]} & {[127,256,538]} & {[63,554]} & {[508,562]}\end{array}$

Pos.: [465]; long-term X-rays: [559, 562]; X-ray off state: [554]; X-ray spectrum: [588, 765]; opt. spectrum: [123, 465, 688]; long-term opt. variations: [127, 256]; $v_{\mathrm{r}} \sin i \sim 600 \mathrm{~km} \mathrm{~s}^{-1}$ : [538]; system velocity: [721]; IR obs.: [232].

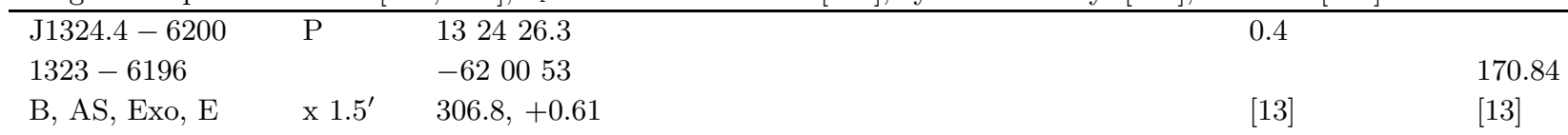

Pos.: [13]; distance > 3.4 kpc: [13]; hard X-ray spe.: [13]; likely a Be/X-ray system: [13].

\begin{tabular}{lllllll}
\hline $1417-624$ & PT & 141725.5 & ${ }^{*} 7$ & $17.2:, 0.7: 1.7,0.8$ & $2-43$ & 42.12 \\
& & -622811 & & OBe, $2:$ & 17.6 \\
U, M, A, S, C & o & $313.0,-1.6$ & {$[18]$} & {$[244]$} & {$[63]$} & {$[207,508]$}
\end{tabular}

Pos.: [63]; X-ray obs.: [18, 207, 364]; opt. id: [244]; orbital parameters: [207]; Centaurus $1971-2$ transient (?): [115, 755]; 1994 II-type outburst: [207].

$\left.\begin{array}{lllll}\hline \text { J1452.8-5949 } & \mathrm{P} & 145249.3 & 0.04 & 437.4 \\ & & -594918 & & {[522]}\end{array}\right]$

Pos.: [522]; distance: [522]; probably a Be star: [522]; X-ray spe.: [522]. 
Table 1. continued

\begin{tabular}{|c|c|c|c|c|c|c|}
\hline Name(s) & $\begin{array}{l}\text { type } \\
\text { Pos. }\end{array}$ & $\begin{array}{l}\mathrm{RA} \\
\mathrm{DEC} \\
l^{\mathrm{II}}, b^{\mathrm{II}}\end{array}$ & Opt. Ctp. & $\begin{array}{l}V, B-V, U-B \\
\text { Sp. type, } E_{B-V}\end{array}$ & $\begin{array}{l}F_{x} \\
\mu \mathrm{Jy}\end{array}$ & $\begin{array}{l}P_{\text {orb }}(\mathrm{d}) \\
P_{\text {pulse }}(\mathrm{s})\end{array}$ \\
\hline $1538-522$ & $\mathrm{P}$ & $\begin{array}{l}153838.6 \\
-521337\end{array}$ & $\begin{array}{l}\text { QV Nor } \\
{ }^{*} 12\end{array}$ & $\begin{array}{l}14.4,1.9,0.6 \\
\text { B0 Iab, } 2.1\end{array}$ & $<3-30$ & $\begin{array}{l}3.73 \\
529\end{array}$ \\
\hline $\mathrm{U}, \mathrm{M}, \mathrm{A}, \mathrm{H}, \mathrm{S}$ & o & $327.4,+2.1$ & {$[17]$} & {$[147,324,537]$} & {$[63]$} & {$[114,508]$} \\
\hline
\end{tabular}

Pos.: [63]; X-ray obs.: [114, 150, 442, 592]; X-ray eclipses: [35, 159]; X-ray orbit: [442]; X-ray spectrum: [442, 765]; X-ray cyclotron line: [94]; long-term spin-up trend: [604]; opt. light curve: [529]; radial-velocity curve: [147, 583]; $v_{\mathrm{r}} \sin i \sim 200 \mathrm{~km} \mathrm{~s}{ }^{-1}$ : [147]; system velocity: [721]; opt. obs.: [537].

\begin{tabular}{|c|c|c|c|c|}
\hline $1553-542$ & $\mathrm{P}$ & 155355.6 & 27 & 30.6 \\
\hline & & -541615 & & 9.3 \\
\hline S & x $3.5^{\prime \prime}$ & $327.9,-0.9 \quad[17]$ & {$[63]$} & {$[367,508]$} \\
\hline
\end{tabular}

Pos.: [63]; opt. counterpart likely a Be star: [367]; X-ray orbit: [367].

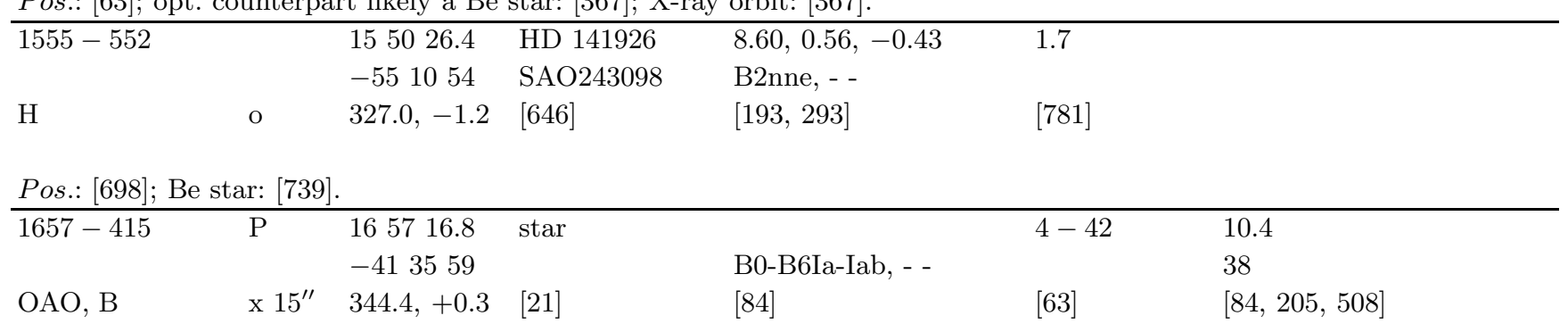

Pos.: [21]; error box $\sharp 2$ : [21, 476, 540]; V861 Sco not the optical counterpart: [21]; X-ray obs.: [358]; X-ray spectrum: [765]; hard X-ray obs.: [476]; orbital solution: [84].

\begin{tabular}{llllll}
\hline $1700-377$ & 170032.7 & HD 153919 & $6.6,0.27,-0.72$ & $<11-110$ & 3.41 \\
& -374629 & V884 Sco & O6.5f, 0.52 & & \\
U, M, S, Gro, C, o & $347.8,+2.2$ & {$[351]$} & {$[259,263,547,778]$} & {$[63]$} & {$[67,251,728]$} \\
B & & & &
\end{tabular}

Pos.: [63]; X-ray variability: [38, 175, 252]; hard X-ray spectrum: [211, 552]; no X-ray pulsations: [175, 236]; opt. light curve: [26, 91, 728]; opt. spectrum: [117, 155, 192, 274]; radial-velocity curve: [175, 258]; $v_{\mathrm{r}} \sin i \sim 140-300 \mathrm{~km} \mathrm{~s}^{-1}:[117,155,305$, 778]; system velocity: [721]; UV obs.: [182, 259, 263, 359]; soft X-ray Raman scattering: [359]; opt. polarimetry: [172, 173]; IR obs.: [232, 547]; system parameters: [270]; polarization: [779]; possibly 13.81 days period: [384].

\begin{tabular}{|c|c|c|c|c|}
\hline \multirow[t]{2}{*}{$\mathrm{J} 170006-4157$} & $\mathrm{P}$ & 17005.3 & 0.4 & \\
\hline & & -415744 & & 714.5 \\
\hline AS & $\mathrm{x} 1^{\prime}$ & $344.0,+0.25$ & {$[691]$} & {$[691]$} \\
\hline
\end{tabular}

Pos.: [691]; can't completely exclude the possibility that it is a WD binary: [691].

\begin{tabular}{lllll}
\hline $1722-363$ & $\mathrm{P}$ & 172233 & $0.2-5$ & \\
& & -362205 & & 413 \\
Exo & $\mathrm{x} 9^{\prime}$ & $351.5,-0.6$ & {$[672,676]$} & {$[508,676]$}
\end{tabular}

Pos.: [744]; hard X-ray spectrum: [676]; limits on orbital period: [672].

\begin{tabular}{llll}
\hline J1739-302 & $\mathrm{T}$ & 173853 & 136 \\
& & -3015.6 & $(2-25 \mathrm{keV})$ \\
$\mathrm{X}$ & $\mathrm{x} 3^{\prime}$ & $358.1,+0.56$ & {$[645]$}
\end{tabular}

Pos.: [645]; radio obs.: [283]; probably a Be/X-ray sys.: [645]; X-ray spec. and short X-ray outburst: [645].

\begin{tabular}{lrl}
\hline J1739.5-2942 & 173930.1 \\
& -294207 \\
$\mathrm{X}, \mathrm{C}$ & $\mathrm{x} 19.2^{\prime \prime}$ & $358.6,-0.74[501]$
\end{tabular}

Pos.: [501]; likely a Be/X-ray binary: [501]; hard X-ray spec.: [501]; identical X-ray source 1736 - 297(?): [501]. 
Table 1. continued

\begin{tabular}{lllllll}
\hline Name(s) & type & RA & Opt. Ctp. & $V, B-V, U-B$ & $F_{x}$ & $\begin{array}{l}P_{\text {orb }}(\mathrm{d}) \\
P_{\text {pulse }}(\mathrm{s})\end{array}$ \\
& & DEC & & Sp. type, $E_{B-V}$ & $\mu \mathrm{Jy}$ & \\
& Pos. & $l^{\mathrm{II}}, b^{\mathrm{II}}$ & {$[\mathrm{FC}]$} & & & \\
\hline J1744.7-2713 & & 174445.4 & HD 161103 & $8.4,0.44,-0.64$ & 0.06 & \\
& & -271347 & & B2V-IIIe & & \\
$\mathrm{R}$ & $\mathrm{x} 18^{\prime \prime}$ & $1.36,+1.1$ & & & &
\end{tabular}

Pos.: [500]; strong emission lines: [500]; distance 0.8 kpc: [500]; likely Be/X-ray binary: [500].

\begin{tabular}{lllll}
\hline J1749.2-2725 & TP & 174910.1 & 1.36 & \\
& & -272516 & & 220.38 \\
AS & x $1^{\prime}$ & $1.7,+0.1$ & {$[690]$} & {$[690]$}
\end{tabular}

Pos.: [690]; weak X-ray source: [690]; hard X-ray spe.: [690]; likely a NS: [690]; within error of a nearby WD: [690].

\begin{tabular}{lllll}
\hline J1750-27 & $\mathrm{P}$ & 174912.0 & 27 & 29.8 \\
& & -263850 & & 4.45 \\
$\mathrm{C}$, AS & $\mathrm{x} 2^{\prime}$ & $2.4,+0.5$ & {$[777]$} & {$[176,627]$}
\end{tabular}

Pos.: [176]; no optical counterpart reported: [627]; likely Be system: [627]; large spin-up: [627].

\begin{tabular}{llll}
\hline $1807-10$ & 1807.9 & $<2-10$ \\
& & -1053 & \\
$\mathrm{U}$ & $\mathrm{T}$ & $1.3^{\circ}$ & $18.6,+3.9$
\end{tabular}

Pos.: (large error box): [215]; uncertain if HMXB or LMXB.

\begin{tabular}{lllll}
\hline J1820.5-1434 & $\mathrm{P}$ & 182029.5 & 1 & \\
& & -143424 & & 152.26 \\
$\mathrm{AS}$ & $\mathrm{x} 0.5^{\prime}$ & $16.5,+0.07$ & {$[376]$} & {$[376]$}
\end{tabular}

Pos.: [376]; a highly obscured accretion-driven binary X-ray: [376].

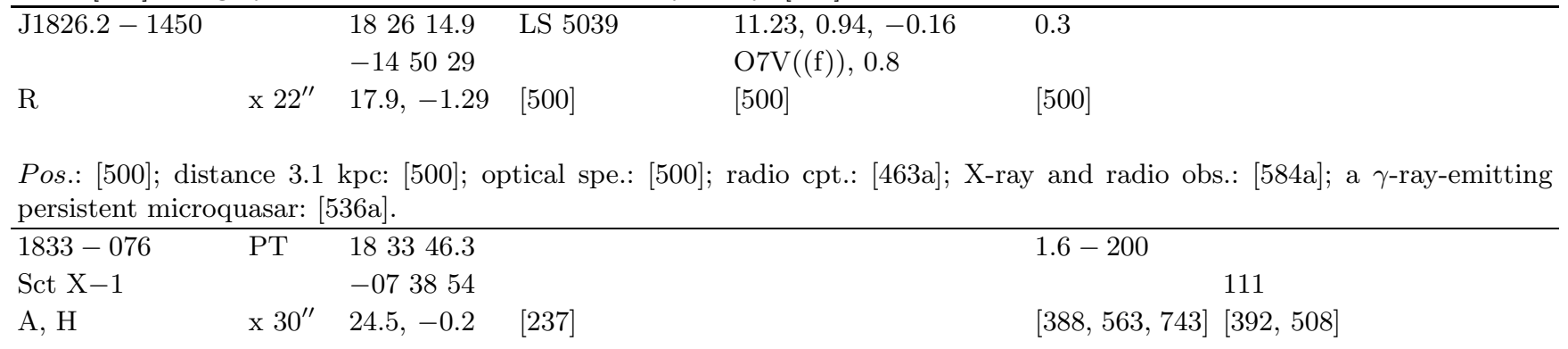

Pos.: [574]; alternative position (18 34 49.5, -07 38 05): [574]; X-ray obs.: [88, 278]; X-ray pulsations: [392]; hard X-ray spectrum: [122, 574]; long-term X-ray record: [563]; transient: [122].

\begin{tabular}{llll}
\hline $1839-06$ & 1839.0 & 1 \\
& $\mathrm{~T}$ & -05.9 & \\
$\mathrm{G}$ & $\mathrm{x} 30^{\prime}$ & $26.6,-0.5$ & {$[388]$}
\end{tabular}

Pos.: [388].

\begin{tabular}{lllll}
\hline $1839-04$ & PT & 1839.2 & 2.5 & \\
& & -04.5 & & 81.1 \\
$\mathrm{G}$ & $\mathrm{x} 24^{\prime}$ & $27.9,+0.1$ & {$[388]$} & {$[388]$}
\end{tabular}

Pos.: [388].

\begin{tabular}{lllll}
\hline $1843+009$ & PT & 1843.0 & $<0.4-33$ & \\
& & +0.9 & & 29.5 \\
G, C, X, B & x $10^{\prime}$ & $33.1,+1.7$ & {$[388]$} & {$[388]$}
\end{tabular}

Pos.: [388]; X-ray obs.: [388, 389]. 
Table 1. continued

\begin{tabular}{|c|c|c|c|c|c|c|}
\hline Name(s) & $\begin{array}{l}\text { type } \\
\text { Pos. }\end{array}$ & $\begin{array}{l}\mathrm{RA} \\
\mathrm{DEC} \\
l^{\mathrm{II}}, b^{\mathrm{II}}\end{array}$ & $\begin{array}{l}\text { Opt. Ctp. } \\
{[\mathrm{FC}]}\end{array}$ & $\begin{array}{l}V, B-V, U-B \\
\text { Sp. type, } E_{B-V}\end{array}$ & $\begin{array}{l}F_{x} \\
\mu \mathrm{Jy}\end{array}$ & $\begin{array}{l}P_{\text {orb }}(\mathrm{d}) \\
P_{\text {pulse }}(\mathrm{s})\end{array}$ \\
\hline $1845-03$ & $\mathrm{~T}$ & $\begin{array}{l}1844.7 \\
-03.2\end{array}$ & & & 1 & \\
\hline $\begin{array}{l}\text { G } \\
\text { Pos.: [388]. }\end{array}$ & x $24^{\prime}$ & $29.7,-0.5$ & & & {$[388]$} & \\
\hline $1845-024$ & $\mathrm{PT}$ & $\begin{array}{l}184541.1 \\
-022837\end{array}$ & & & $1-44$ & $\begin{array}{l}241 \\
94.8\end{array}$ \\
\hline $\mathrm{A}, \mathrm{H}, \mathrm{S}, \mathrm{G}, \mathrm{C}, \mathrm{B}$ & $\mathrm{x} 30^{\prime \prime}$ & $30.4,-0.4$ & {$[237]$} & & {$[63,388]$} & {$[792,388]$} \\
\hline
\end{tabular}

Pos.: [63]; X-ray obs.: [178, 388, 390, 630]; distance: [792]; same sources as GS 1843-02, X1845 - 024, and GRO J1849-03: [647]; likely a Be/X-ray: [647, 209]; X-ray outburst: [209]; high eccentricity (0.88): [209].

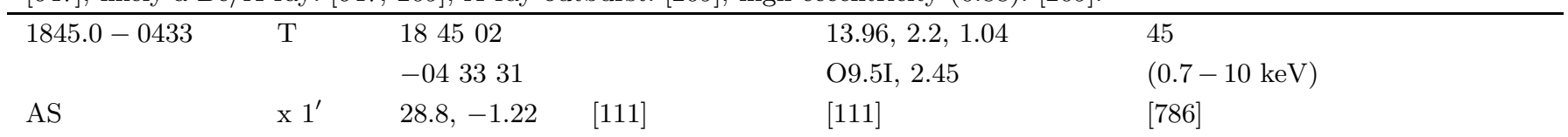

Pos.: [111]; discovery X-ray source: [786]; optical spectrum: [111]; distance 3.6 kpc: [111].

\begin{tabular}{|c|c|c|c|c|}
\hline $1855-02$ & $\mathrm{PT}$ & 1855.4 & \multicolumn{2}{|l|}{2} \\
\hline & & -02.8 & & \\
\hline G & x $10^{\prime}$ & $31.3,-2.7$ & \multicolumn{2}{|l|}{ [388] } \\
\hline \multicolumn{5}{|l|}{ Pos.: [388]. } \\
\hline \multirow[t]{2}{*}{ J1855-026 } & $\mathrm{P}$ & 1855.7 & 6 & 6.1 \\
\hline & & -0237 & & 361 \\
\hline $\mathrm{X}$ & $\times 6^{\prime}$ & $31.1,-2.15$ & {$[460]$} & {$[134,460]$} \\
\hline
\end{tabular}

Pos.: [460]; most likely a SG/X-ray system: [134]; BD-2 4786 not the opt. cpt.: [134]; X-ray spe.: [134].

\begin{tabular}{|c|c|c|c|c|}
\hline \multirow{2}{*}{ J1858+034 } & $\mathrm{TP}$ & 1858.6 & 25 & \\
\hline & & 0321 & & 221.0 \\
\hline $\mathrm{X}$ & $\mathrm{x} 2.5^{\prime}$ & $36.7,-0.07$ & {$[582]$} & [670] \\
\hline
\end{tabular}

Pos.: [462]; QPO: [544]; probably a Be/X-ray sys.: [670]; very hard X-ray spec.: [462,544].

\begin{tabular}{|c|c|c|c|}
\hline \multirow[t]{3}{*}{$1901+03$} & $\mathrm{~T}$ & 1901.7 & $<2-87$ \\
\hline & & +0306.0 & \\
\hline & x $10^{\prime}$ & $37.2,-1.4$ & {$[115]$} \\
\hline
\end{tabular}

Pos.: [215]; transient 1971: [115, 214]; hard X-ray spectrum: [115].

\begin{tabular}{|c|c|c|c|c|}
\hline \multirow[t]{2}{*}{ J1906+09 } & $\mathrm{P}$ & 190520 & 0.7 & \\
\hline & & 0902.5 & & 89.17 \\
\hline $\mathrm{X}$ & $\times 2^{\prime}$ & $42.6,+1.04$ & {$[457]$} & {$[457]$} \\
\hline
\end{tabular}

Pos.: [667]; distance: [457]; likely a supergiant: [457]; not associated with the soft gamma-ray repeater SGR 1900+14: [671]; X-ray spec.: [457].

$\begin{array}{lllllll}1907+097 & \text { PT } & 190715.1 & \text { star } & 16.4,3.2,-- & 4-275 & 8.38 \\ & & +094454 & & \text { B I, 3.3 } & & 438 \\ \mathrm{U}, \mathrm{M}, \mathrm{A}, \mathrm{H} & \mathrm{o} & 43.7,+0.5 & {[625]} & {[625,718]} & {[63]} & {[441,508]}\end{array}$

Pos.: [625]; long-term X-ray recod: [563]; 1980 outburst: [459]; orbital parameters: [120, 441]; orbital modulation X-ray flux: [459]; opt. spectrum: [337, 718]; $v_{\mathrm{r}} \sin i \sim 85 \mathrm{~km} \mathrm{~s}^{-1}:[718]$. 
Table 1. continued

\begin{tabular}{|c|c|c|c|c|c|c|}
\hline Name(s) & $\begin{array}{l}\text { type } \\
\text { Pos. }\end{array}$ & $\begin{array}{l}\text { RA } \\
\mathrm{DEC} \\
l^{\mathrm{II}}, b^{\mathrm{II}}\end{array}$ & Opt. Ctp. & $\begin{array}{l}V, B-V, U-B \\
\text { Sp. type, } E_{B-V}\end{array}$ & $\begin{array}{l}F_{x} \\
\mu \mathrm{Jy}\end{array}$ & $\begin{array}{l}P_{\text {orb }}(\mathrm{d}) \\
P_{\text {pulse }}(\mathrm{s})\end{array}$ \\
\hline $1909+048$ & & $\begin{array}{l}190921.3 \\
+045354\end{array}$ & $\begin{array}{l}\text { SS433 } \\
\text { V1343 Aql }\end{array}$ & $\begin{array}{l}14.2,2.1,0.6 \\
\text { pec, } 2.6\end{array}$ & $2-10$ & 13.1 \\
\hline $\mathrm{U}, \mathrm{A}, \mathrm{H}, \mathrm{G}$ & $\mathrm{o}, \mathrm{r}$ & $39.7,-2.2$ & {$[407]$} & {$[450,507,740]$} & [63] & {$[145]$} \\
\hline
\end{tabular}

Pos.: [63]; X-ray obs.: [30, 38, 68, 69, 245, 363]; Doppler shift X-ray Fe line: [468, 751]; extended X-ray lobes: [631, 750]; $\gamma$-ray obs.: [223, 398]; Doppler shifted opt. emission lines: [448, 451]; stationary emission lines from accretion disk: [204]; kinematic model precessing (164 d) high-speed jets: [6]; opt. spe.: [24, 191, 194, 386, 452]; opt. spectrophot.: [9, 385, 740]; opt. lt. curve: [14, 15, 23, 275, 406, 408, 409]; long-term optical phot. record: [275, 369, 469]; X-ray/opt. eclipse: [658]; IR obs.: [382, 742]; precession clock: [10]; radio structure: [31, 197, 198, 596, 651, 732]; radio variability: [57, 202]; distance 5.5 kpc: [284]; nature of the compact star: [171]; reviews: [447, 731, 793]; circularly polarized radio emissiom: [200]; VLBA multifreq. obs.: [531].

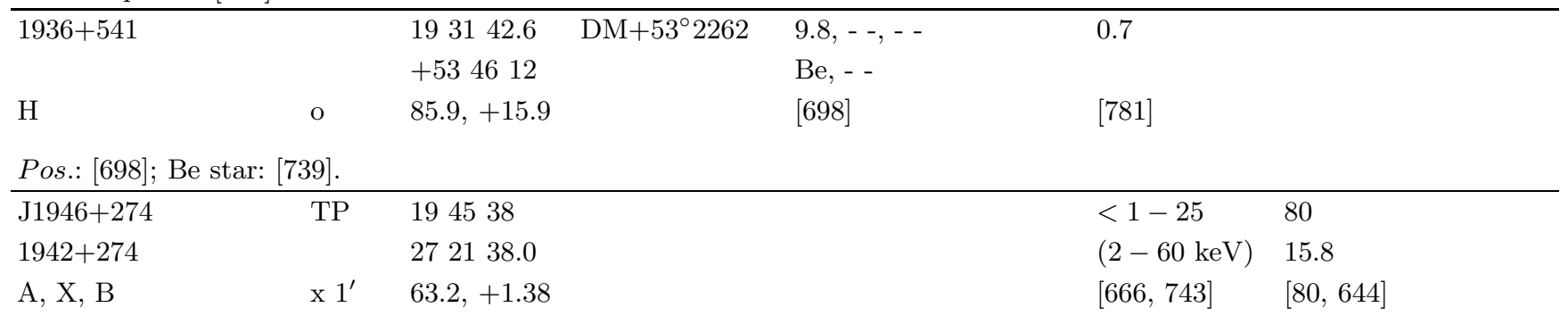

Pos.: [80]; transient Nov.-Dec. 1976: [743]; X-ray flare: [80]; likely a Be: [80]; very hard X-ray spec.: [644]; same as 3A $1942+274$ and 1SAX J1946.5+2721: $[80,666]$.

\begin{tabular}{llllll}
\hline $1947+300$ & $\mathrm{~T}$ & 194736.3 & $* 3$ & $14.2,0.9,-0.3$ & $<10-84$ \\
& +300454 & &,-- 1.1 & \\
$\mathrm{~K}$ & $\mathrm{o}$ & $66.1,+2.1$ & {$[238]$} & {$[234,238]$} & {$[60]$}
\end{tabular}

Pos.: [238]; H $\alpha$ emission: [234]; likely, but not certain this is a HMXB: [234, 238]; see also [638].

\begin{tabular}{lllcl}
\hline J1948+32 & $\mathrm{P}$ & 1948 & $27-53$ & $35-70$ \\
& & 32.0 & $(20-75 \mathrm{keV})$ & 18.7 \\
$\mathrm{C}$ & $\mathrm{x}$ & $64.9,+1.8$ & {$[85]$} & {$[85]$}
\end{tabular}

Pos.: [85]; eccentricity < 0.25: [85]; mass function: [85]; probably a Be: [85].

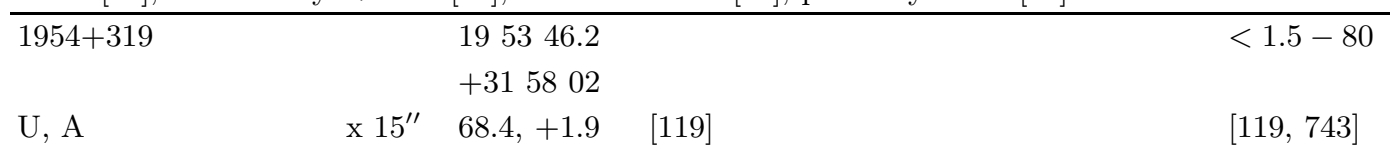

Pos.: [186]; heavily reddened supergiant system (?): [699].

\begin{tabular}{lllllll}
\hline $1956+350$ & U & 195628.9 & HD 226868 & $8.9,0.84,-0.26$ & $235-1320$ & 5. \\
Cyg X-1 & & +350355 & V1357 Cyg & O9.7 Iab, 1.06 & & \\
U, M, A, H, X, G, C & o, r & $71.3,+3.1$ & {$[568]$} & {$[116,158,518]$} & {$[63]$} & {$[227]$}
\end{tabular}

Pos.: [63]; hard X- and $\gamma$-ray obs.: [226, 419, 608, 626, 700, 701]; long-term X-ray obs.: [289, 565]; X-ray low/high states: [418, 674]; X-ray dips: [29, 378, 581]; rapid X-ray variability: [37, 38, 427, 471, 486, 487, 488, 602]; QPO: [218, 387, 735, 736]; chaotic (?): [426, 707]; 300 d period: [228, 368, 370, 565]; X-ray spectrum: [32, 33, 189, 379, 432, 737, 759]; $\gamma$-ray obs.: [420, 551]; radio obs.: [65, 66, 281, 286]; opt. spectra: [1, 2, 116, 229, 519, 648]; radial-velocity curve: [227, 228, 519]; $v_{\mathrm{r}} \sin i \sim 100 \mathrm{~km} \mathrm{~s}^{-1}:$ [229]; system velocity: [721]; nature of the compact star: [52, 633, 752]; opt. light curve: [370, 435]; opt. UV polarimetry: [174, 217, 779]; UV obs.: [158, 182, 692, 783]; IR obs.: [402]; reviews (1977): [521]; fund. parameters: [277].

\begin{tabular}{|c|c|c|c|c|c|c|}
\hline $2030+375$ & PT & 203022.1 & ${ }^{*} 2$ & $19.7,3.3,--$ & $<0.5-1400$ & 46.0 \\
\hline Exo, C, B, X, AS & о $2^{\prime \prime}$ & $\begin{array}{l}+372800 \\
77.2,-1.3\end{array}$ & {$[107,496]$} & $\begin{array}{l}\text { B0, 3.8 } \\
{[107,496]}\end{array}$ & {$[541]$} & $\begin{array}{l}41.8 \\
{[508,541,662]}\end{array}$ \\
\hline
\end{tabular}

Pos.: [496]; outburst: [541]; rapid X-ray variability: [38]; $0.2 \mathrm{~Hz}$ QPO: [12]; opt. spe.: [107, 342]; long-term opt./IR variability: [580]. 
Table 1. continued

\begin{tabular}{lllllll}
\hline Name(s) & type & RA & Opt. Ctp. & $V, B-V, U-B$ & $F_{x}$ & $P_{\text {orb }}(\mathrm{d})$ \\
& & DEC & & Sp. type, $E_{B-V}$ & $\mu \mathrm{Jy}$ & $P_{\text {pulse }}(\mathrm{s})$ \\
& Pos. & $l^{\mathrm{II}}, b^{\mathrm{II}}$ & {$[\mathrm{FC}]$} & & \\
\hline $2030+407$ & & 203037.6 & V1521 Cyg & $I=20.0$ & $90-430$ & 0.20 \\
Cyg X-3 & & +404713 & & Wolf-Rayet, 6.3 & & {$[543]$} \\
U, M, A, H, CX & $\mathrm{r}$ & $79.9,+0.7$ & {$[741]$} & {$[493,719,741]$} & {$[63]$}
\end{tabular}

Pos.: [63]; distance: [168]; orb. period change: [380, 712]; long-term X-ray obs.: [289, 564]; no X-ray pulsations: [380, 782]; orbital X-ray curve: [55, 377]; aperiodic variability: [38]; X-ray halo: [490]; transient QPO: [713]; radio outbursts: [241, 282, 346, 492]; quiescent radio flares (period 4.95 h?): [491]; radio jet \& lobes: [55, 222, 664]; IR phot.: [36, 464, 466]; IR spectra (secondary helium star): [362, 719]; no X-ray pulsations: [782]; X-ray spectrum: [377, 758]; 1989 review (incl. TeV/PeV $\gamma$-rays: [53]; spe. variability during outburst and quiescence: [199]; VLBI obs. during outburst: [615].

\begin{tabular}{|c|c|c|c|c|c|}
\hline $\mathrm{J} 2030.5+4751$ & & 203030.6 & $\mathrm{SAO} 49725$ & $9.27,0.38,-0.65$ & 0.04 \\
\hline $\mathrm{R}$ & $\mathrm{x} 18^{\prime \prime}$ & $\begin{array}{l}475146 \\
85.2,+5.02\end{array}$ & [500] & $\begin{array}{l}\text { B0.5V-IIIe, - - } \\
{[500]}\end{array}$ & [500] \\
\hline
\end{tabular}

Pos.: [500]; strong emission lines: [500]; distance 2.2 kpc: [500]; likely Be/X-ray binary: [500].

\begin{tabular}{|c|c|c|c|c|}
\hline \multirow[t]{2}{*}{$\mathrm{J} 2058+42$} & $\mathrm{TP}$ & 2059.0 & 320 & 110 \\
\hline & & 4143 & & 198 \\
\hline $\mathrm{C}, \mathrm{X}$ & $\times 4^{\prime}$ & $83.5,-2.75$ & {$[775]$} & {$[775]$} \\
\hline
\end{tabular}

Pos.: [775]; orbital period 54 days: [129]; giant outburst in 1995: [775]; most likely a Be/X-ray sys.: [775]; one of two stars likely opt. ctp.: [83].

\begin{tabular}{lllll}
\hline J2103.5+4545 & $\mathrm{P}$ & 210333 & 20 & 12.68 \\
& & 4545.0 & 358.61 \\
$\mathrm{~B}, \mathrm{X}$ & $\mathrm{x} 2.5^{\prime}$ & $87.1,+0.71$ & {$[34]$} & {$[34,301]$}
\end{tabular}

Pos.: [301]; distance 4 kpc: [34]; eccentricity 0.4: [34]; HD 200709 not opt. cpt.: [34]; X-ray spe.: [301].

$\begin{array}{llllll}2138+568 & \text { PT } & 2138.0 & \text { star } & & <6-100 \\ \text { Cep X-4? } & & +5650.0 & & \text { B1 } & 66.2 \\ \text { M, H, G } & \text { x } 7^{\prime} & 99.0,+3.3 & {[53 a]} & {[391]} & {[391,508]}\end{array}$

Pos.: [391]; likely Be/X-ray system: [391]; X-ray obs.: [391]; X-ray cyclotron line: [481]; same as Cep X-4(?): [454, 704]; X-ray outburst: [776]; orbital period $23-147.3$ days: [776].

\begin{tabular}{|c|c|c|c|c|c|}
\hline $2202+501$ & & $\begin{array}{l}215944.1 \\
+495535\end{array}$ & $\begin{array}{l}\mathrm{DM}+49^{\circ} 3718 \\
\mathrm{SAO} 51568\end{array}$ & $\begin{array}{l}8.8,--,-- \\
\text { Be, - - }\end{array}$ & 0.7 \\
\hline $\mathrm{H}$ & o & $97.3,-4.0$ & {$[646]$} & [698] & [781] \\
\hline
\end{tabular}

Pos.: [698]; Be star: [739].

\begin{tabular}{|c|c|c|c|c|c|c|}
\hline \multirow[t]{2}{*}{$2206+543$} & & 220607.4 & star & $9.9,0.2,-0.6$ & $0.6-5.5$ & \\
\hline & & +541623 & & B1e, 0.5 & & $392(?)$ \\
\hline $\mathrm{U}, \mathrm{M}, \mathrm{A}, \mathrm{H}$ & o $2^{\prime \prime}$ & $100.6,-1.1$ & {$[654]$} & {$[654]$} & {$[743,781]$} & {$[611]$} \\
\hline
\end{tabular}

Pos.: [654]; X-ray observations: [611]; H $\alpha$ emission: [123].

\begin{tabular}{|c|c|c|c|c|c|}
\hline \multirow[t]{2}{*}{$2214+589$} & & 222447.8 & GG3 71 & $11,--,--$ & 0.5 \\
\hline & & +605859 & & $\mathrm{~B}[\mathrm{e}],--$ & \\
\hline $\mathrm{H}$ & o & $106.4,+3.1$ & [233] & {$[264,698]$} & [781] \\
\hline
\end{tabular}

Pos.: [698]; Be star: [739]; Herbig Ae/Be candidate: [264]; associated with IRAS source 22248+6058: [264].

\begin{tabular}{lllllll}
\hline J2239.3+6116 & T & 223920.90 & star & $15.1,1.4,--$ & 16 & 262 \\
& & +611626.8 & & B0V-B2IIIe, 1.4 & & \\
B, A, U, X & $00.3^{\prime \prime}$ & $107.7,+2.3$ & {$[327 \mathrm{a}]$} & {$[327 \mathrm{a}]$} & {$[327 \mathrm{a}]$} & {$[327 \mathrm{a}]$}
\end{tabular}

Pos.: [327a]; distance of 4.4 kpc: [327a]; position coincided with $4 \mathrm{U} 2238+60$ and 3A 2237+608: [327a]. 